\title{
Synthesis of Pyrene-Anthracene Conjugated Molecular Rods
}

\author{
J.G. Domínguez Chávez, S. Hernandez Ortega and M. Martínez-García*
}

Instituto de Química, Universidad Nacional Autónoma de México, Cd. Universitaria, Coyoacán, C.P. 04510, México D.F., México

\begin{abstract}
Fully conjugated pyrene-anthracene-based molecular rods were synthesized by the Horner-WadsworthEmmons reaction utilizing potassium tert-butoxide in dry THF. The synthesized rods, which have butylene groups as solubility spacers in the main chain, exhibited good solubility in polar solvents. The solutions of the synthesized pyrenecontaining molecular rods exhibited a blue shift in the UV-vis from the absorption maximum due to the presence of the pyrene group in comparison with the dialdehydes. The chemical structure and purity of the synthesized rods were confirmed by ${ }^{1} \mathrm{H}$ and ${ }^{13} \mathrm{C} \mathrm{NMR}, \mathrm{FAB}+$, MALDI-TOF, electrospray mass spectra and elemental analysis.
\end{abstract}

Keywords: Molecular rods, Horner-Wadsworth-Emmons, anthracene, pyrene.

The presence of charge-transfer reaction centers in macromolecules depends on factors as the composition, the separation in the interchromophore, the overall dynamical and supramolecular reorganization, and the electronic coupling [1]. The synthesis of artificial charge-transfer model systems in which the introduction of simple molecular changes is used to control and tune the magnitude of the electrontransfer parameters became a theme of great interest in the last years. The molecular chains or bridges inside the molecule provide endless opportunities, since they determine the cal entities in molecular and supramolecular electronic and photonic devices [7]. In this context, the combination of anthracene and styrene with pyrene compounds could lead to systems with good response in the electron-transfer processes [8].

In the present work, we describe the synthesis of a new series of compounds in which pyrene is linked to a vinyl anthracene and oligo(phenylvinylene)anthracene. These conjugated block oligomers with well defined structure and do-

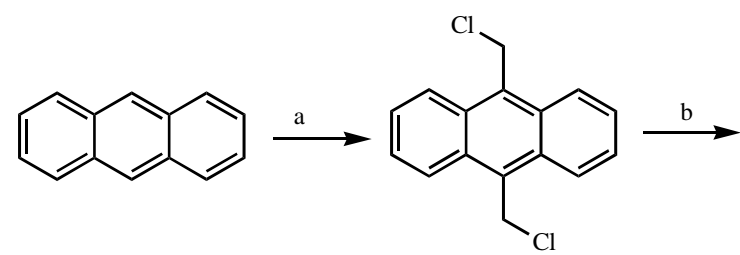

1

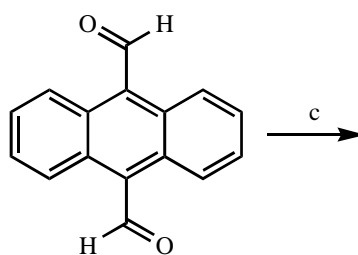

2

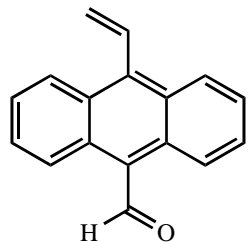

3

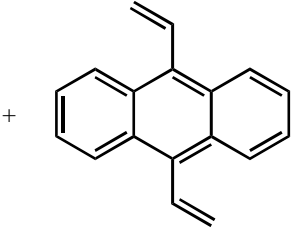

4

Scheme 1. a) 1,4-dixane, $\mathrm{HCl}, \mathrm{NaCl} / \mathrm{H}_{2} \mathrm{SO}_{4}$; b) DMSO, $\mathrm{N}_{2}$, EtOH, 2-nitropropane, $\mathrm{Na}$; c) $\mathrm{CH}_{3}(\mathrm{Ph})_{3} \mathrm{PBr}, n$-BuLi, THF, $\mathrm{N}_{2}$.

structural features and the size of the electronic coupling between the donor and acceptor fragments [2]. The modification of the spacer gives the possibility to introduce a systematic alteration of separation, orientation, and overlap without affecting the electronic nature of the donor-acceptor pair, for which the coupling is proportional to the overlap of their electronic clouds [3]. The electron-transfer processes depend on molecular size and structure, as well as the temperature and the free-energy difference between the donor and acceptor in donor-bridge-acceptor systems. Currently, monodisperse and soluble $\pi$-conjugated systems with a well-defined length and composition are receiving a growing interest for the design of molecular wires [4] and rods [5], since they can be used as tunable building blocks [6] for nanoscale chemi-

*Address correspondence to these authors at the Instituto de Química, Universidad Nacional Autónoma de México, Cd. Universitaria, Coyoacán, C.P. 04510, México D.F., México; E-mail: margar@servidor.unam.mx nor-bridge array were synthesized by Heck and HornerWadsworth-Emmons reactions using aromatic mono vinyl aldehydes, dialdehydes and diphosphonates as monomers.

\section{RESULTS AND DISCUSSION}

The synthesis of the monovinyl and divinylanthracene $\mathbf{3}$ and 4 was started from a chloromethylation of the anthracene to obtain the 9,10-bis(chloromethyl) anthracene $\mathbf{1}$. Treatment of 1 with sodium, 2-nitropropane in DMSO and ethanol gives the anthracene-9,10-dicarbaldehyde $\mathbf{2}$ in good yield (83 $\%$ ). Compound 2 was treated with $\mathrm{CH}_{3}(\mathrm{Ph})_{3} \mathrm{PBr}, n-\mathrm{BuLi}$ in dry THF to obtain compounds 3 and $\mathbf{4}$ (Scheme 1).

The following signals were observed in the ${ }^{1} \mathrm{H}$ NMR spectrum of compound 3: two doublets at $\delta 5.48$ and at $\delta$ 5.97 for the vinylic protons, which coupling constant $J=18$ $\mathrm{Hz}$; one multiplet at 7.25 due to the $=\mathrm{CH}$ group; three signals at $\delta 7.40,7.53,8.22$ and 8.80 due to the aryl group, and, finally, the signal at $\delta 11.30$ assigned to the carboxylic group. 

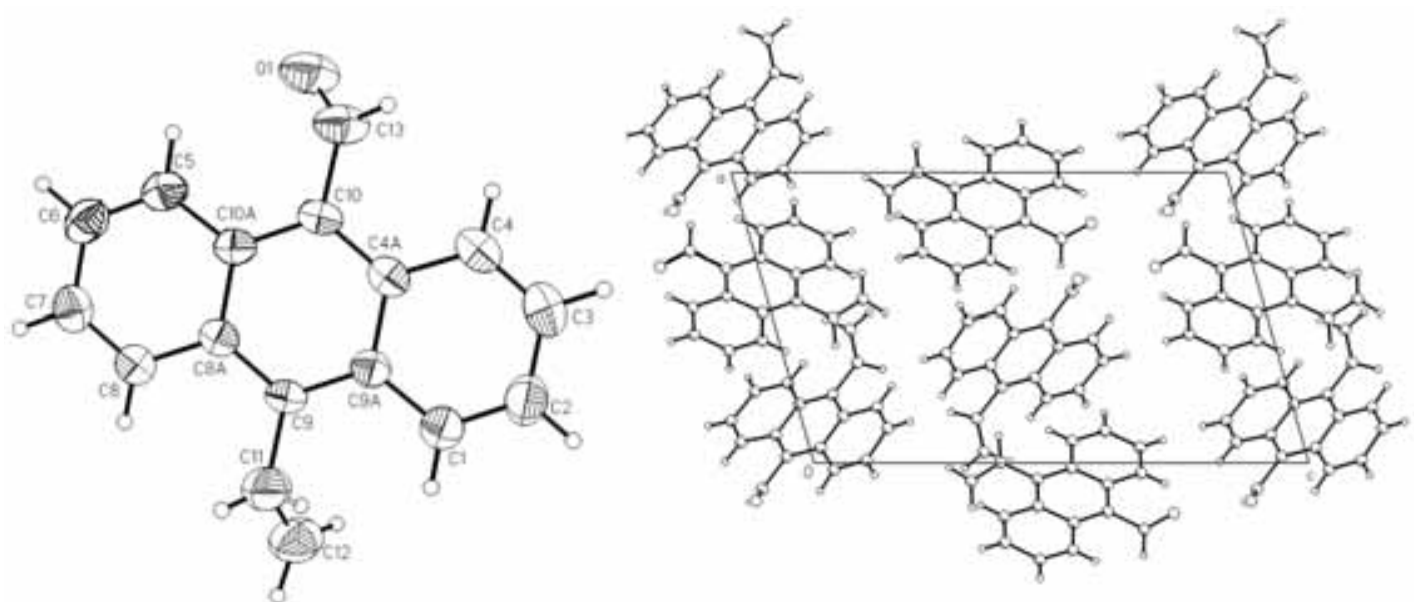

Fig. (1). Crystal structure and crystal packing of the 10-vinylanthracene-9-carbaldehyde 3. Selected bond lengths $[\AA]$ : $\mathrm{O}(1)$-C $(13)=1.162$, $\mathrm{C}(10)-\mathrm{C}(13)=1.471, \mathrm{C}(9)-\mathrm{C}(11)=1.477, \mathrm{C}\left(11-\mathrm{C}(12)=1.206\right.$. Selected angles $\left({ }^{\circ}\right)$ : $\mathrm{C}(10 \mathrm{~A})-\mathrm{C}(10)-\mathrm{C}(13)=121.3, \mathrm{C}(4 \mathrm{~A})-\mathrm{C}(10)-\mathrm{C}(13)=117.9$, $\mathrm{C}(8 \mathrm{~A})-\mathrm{C}(9)-\mathrm{C}(11)=118.7, \mathrm{O}(1)-\mathrm{C}(13)-\mathrm{C}(10)=128.1, \mathrm{C}(12)-\mathrm{C}(11)-\mathrm{C}(9)=118.7$.
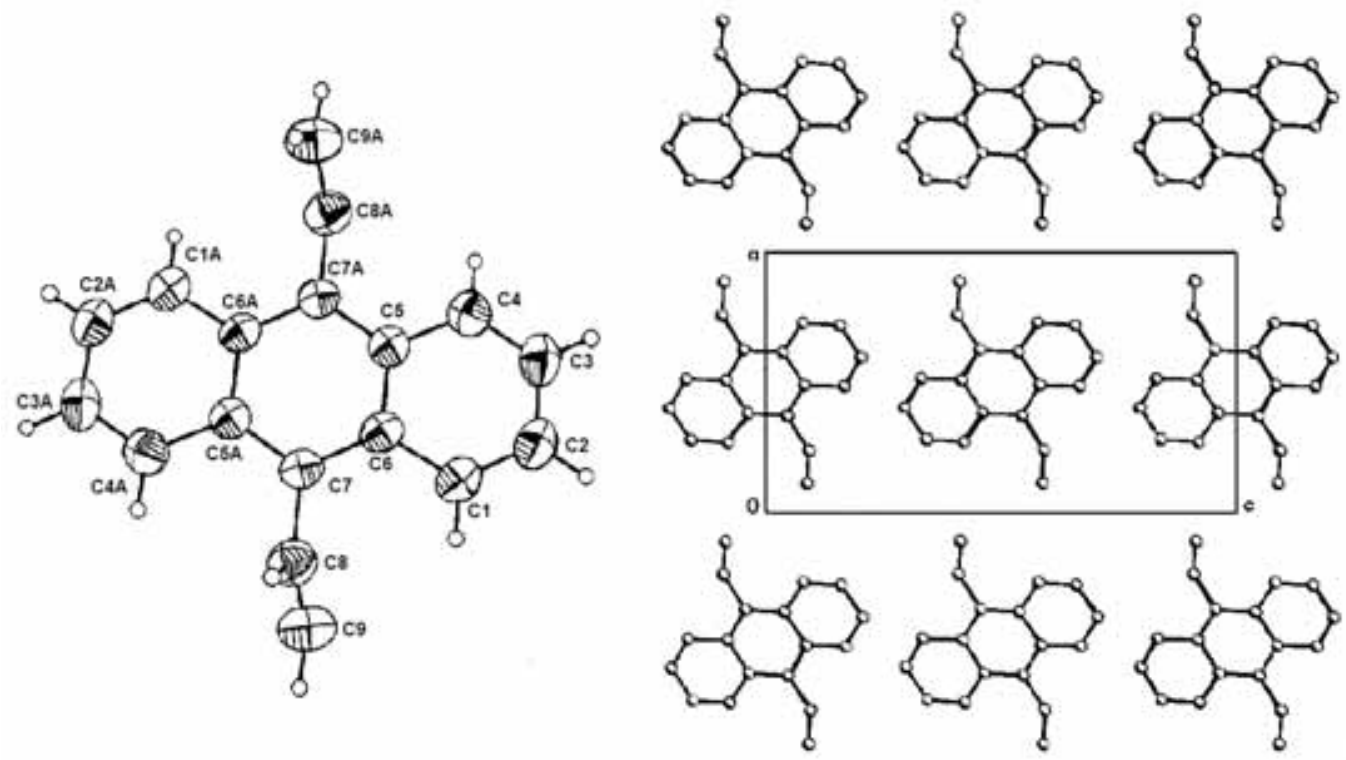

Fig. (2). Crystal structure and crystal packing of the 9,10-divinylanthracene 4. Selected bond lengths $[\AA]$ : $C(8)$-C(9)=1.284, C(7)$\mathrm{C}(8)=1.477$. Selected angles $\left({ }^{\circ}\right)$ : $\mathrm{C}(5)-\mathrm{C}(7)-\mathrm{C}(8)=119.87, \mathrm{C}(9)-\mathrm{C}(8)-\mathrm{C}(7)=126.35$.

The mono and divinyl anthracenes $\mathbf{3}$ and $\mathbf{4}$ were crystallized in dichloromethane and the structures were confirmed by Xray crystallographic studies (Figs. $\mathbf{1}$ and $\mathbf{2}$ ).

In the second step, the synthesis of the counterpart for the oligoanthracenevinylene (OAV) preparation started with the alkylation of the hydroquinone with 1-bromobutane under classical Williamson conditions ( $\mathrm{NaOH}$, ethanol, reflux) to yield the 1,4-dibutoxybenzene 5 in $94 \%$ yield. Halogenation of 5 with bromine in $\mathrm{CCl}_{4}$ afforded 1,4-dibromo-2,5dibutoxybenzene 6 in $85 \%$ yield (Scheme 2).

As 6 has two bromine groups a Mizoroki-Heck cross coupling reaction was carried out with two equivalents of 10-<smiles>CCCCOc1cc(Br)c(OCCCC)cc1Br</smiles>

Scheme 2. Synthesis of 1,4-dibromo-2,5-dibutoxybenzene 6. 


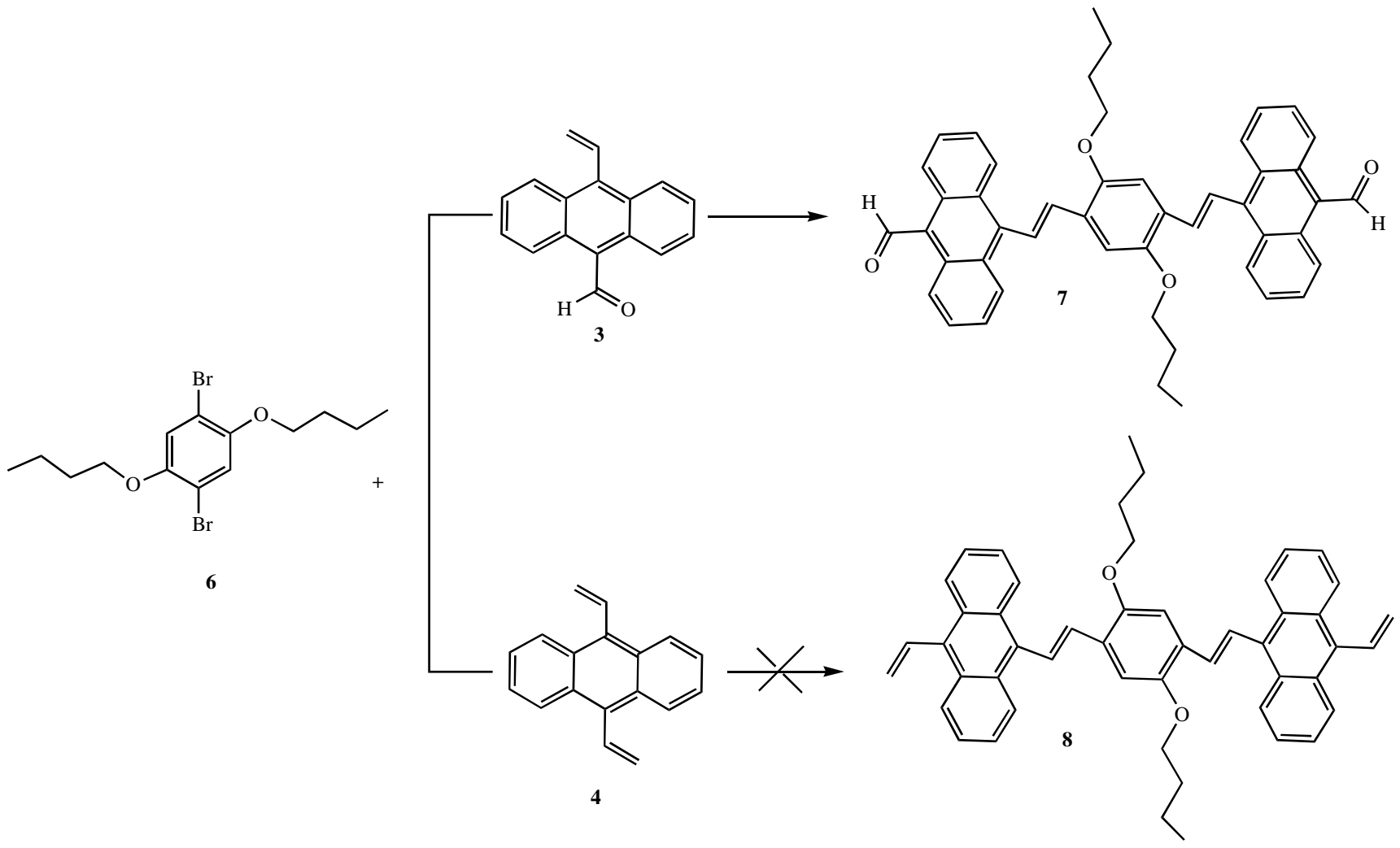

Scheme 3. Synthesis of the dihaldehydes $\mathbf{7}$ and $\mathbf{8}$.

vinylanthracene-9-carbaldehyde $\mathbf{3}$ and 9,10-divinylanthracene 4. For this $\mathrm{Pd}(\mathrm{OAc})_{2}$ was used as catalyst in presence of tri-o-tolylphosphine (TOP) in $\mathrm{DMF}^{-E t}{ }_{3} \mathrm{~N}(1: 1)$ at $110{ }^{\circ} \mathrm{C}$ (Scheme 3). Under these conditions, the bridge compound 7 was obtained in low yield (5\%), and compound 8 was not observed. This could be due to the fact that the central step in the mechanism of the Heck reaction is the syn-addition of the palladium-organic species to the double bond. In the case of 9, 10- divinylanthracene 4 , due to its stereochemistry, the necessary proximity of the insertion compound cannot be achieved. As a result, no addition to the vinylic double bond was observed.

To avoid the problem, the Horner-Wadsworth-Emmons reaction $[9,10]$ was used. For this, the 1,4 dibutoxy benzene 5 was converted to the benzyl choride $\mathbf{9}$. Then, 9 was converted to $\mathbf{1 0}$ via a Michaelis-Arbuzov reaction [11-13] in high yield (Scheme 4).

The Horner-Wadsworth-Emmons reaction was carried out between the anthracene-9,10-dicarbaldehyde $\mathbf{2}$ and $\mathbf{1 0}$ using potassium tert-butoxide in dry THF for $5 \mathrm{~h}$ (Scheme 5).

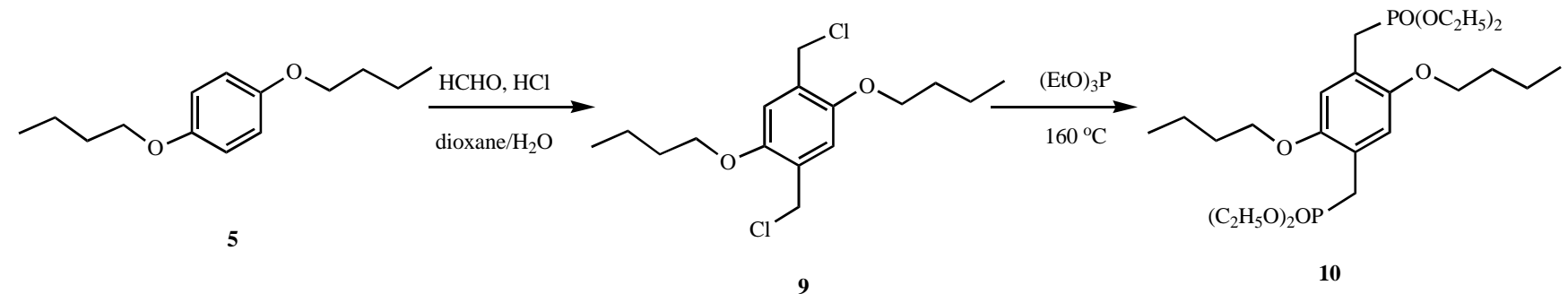

Scheme 4. Synthesis of the tetraethyl (2,5-dibutoxy-1,4-phenylene)bis(methylene) diphosphonate $\mathbf{1 0}$.
Compounds 7 and $\mathbf{1 1}$ were characterized by ${ }^{1} \mathrm{H}$ NMR spectroscopy. In the spectrum of 7 the following signals were observed: one triplet at $\delta_{\mathrm{H}} 0.96$ assigned to the $\mathrm{CH}_{3}$ groups, two multiplets at $\delta_{\mathrm{H}} 1.44$ and at $\delta_{\mathrm{H}} 1.72$ due to the $\mathrm{CH}_{2}$ groups, one triplet at $\delta_{\mathrm{H}} 4.30$ for the $\mathrm{CH}_{2}-\mathrm{O}$, two doublets at $\delta_{\mathrm{H}} 7.54$ and at $\delta_{\mathrm{H}} 7.69$ with a coupling constants $J=16.8$ and $J=17.1 \mathrm{~Hz}$ for the vinylic protons, the characteristic signals at $\delta_{\mathrm{H}} 8.06, \delta_{\mathrm{H}} 8.98$ for the anthracene, and finally one singlet at $\delta_{\mathrm{H}} 11.54$ due to the carbonyl group $\mathrm{HC}=\mathrm{O}$. For compound 11, the most important signal was one doublet due to the ethyl group joined with the phosphonate at $\delta_{\mathrm{H}} 3.33$ with coupling constant $J_{\mathrm{H}-\mathrm{P}}=21.9 \mathrm{~Hz}$. In the ${ }^{31} \mathrm{P}$ NMR spectrum of compound $\mathbf{1 1}$ one singlet was observed at $27.57 \mathrm{ppm}$.

The donor 15 was synthesized from the commercially available 1-pyrenecarboxaldehyde $\mathbf{1 2}$ via the reduction of the aldehyde 12 to obtain the pyren-1-ylmethanol 13 and its chloration with $\mathrm{SOCl}_{2}$ and pyridine to obtain the compound 14 (Scheme 6) and finally, treatment of 14 with triethyl phosphate yielded the compound $\mathbf{1 5}$. 


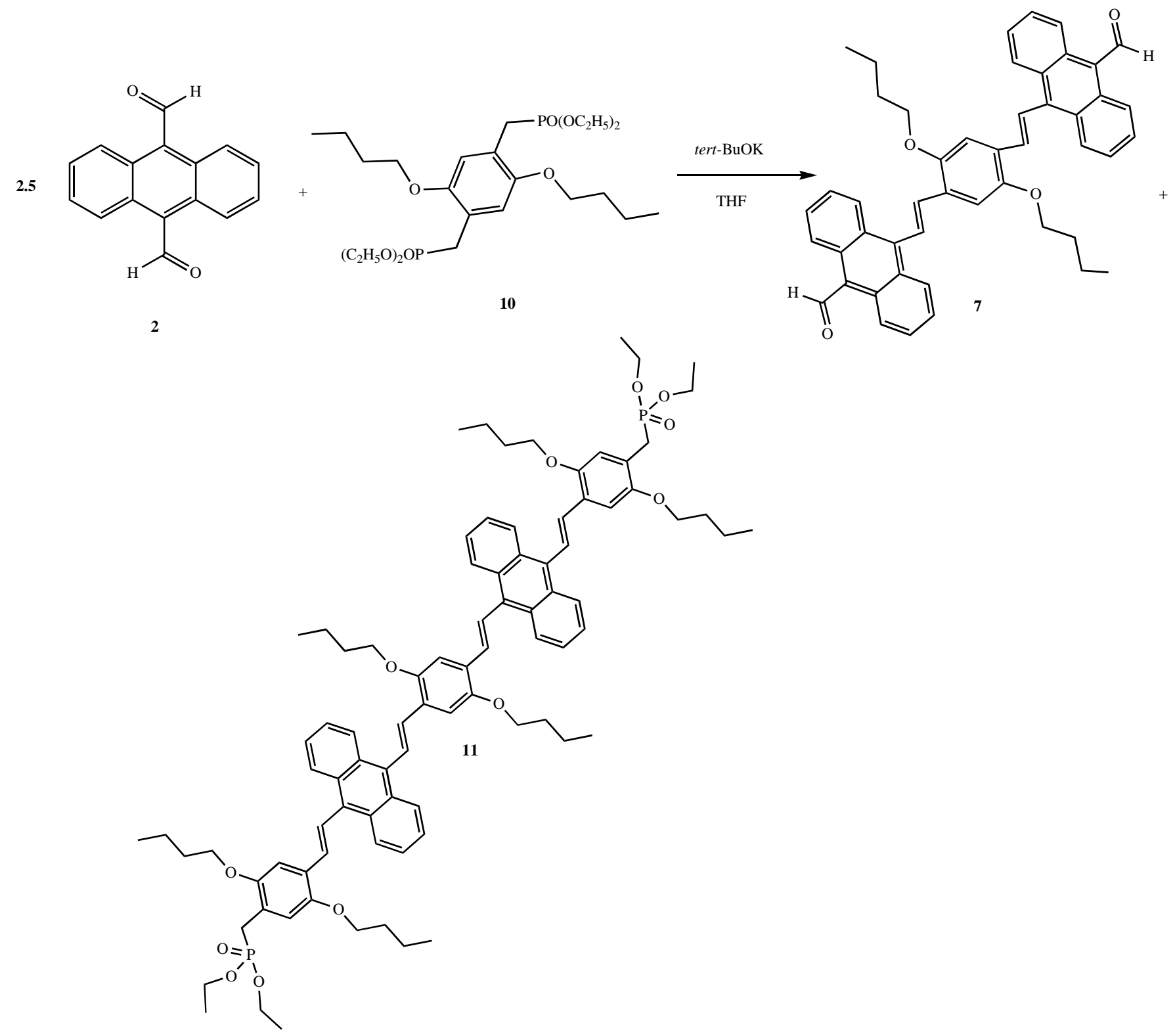

Scheme 5. Synthesis of the dialdehydes 7 and $\mathbf{1 1 .}$

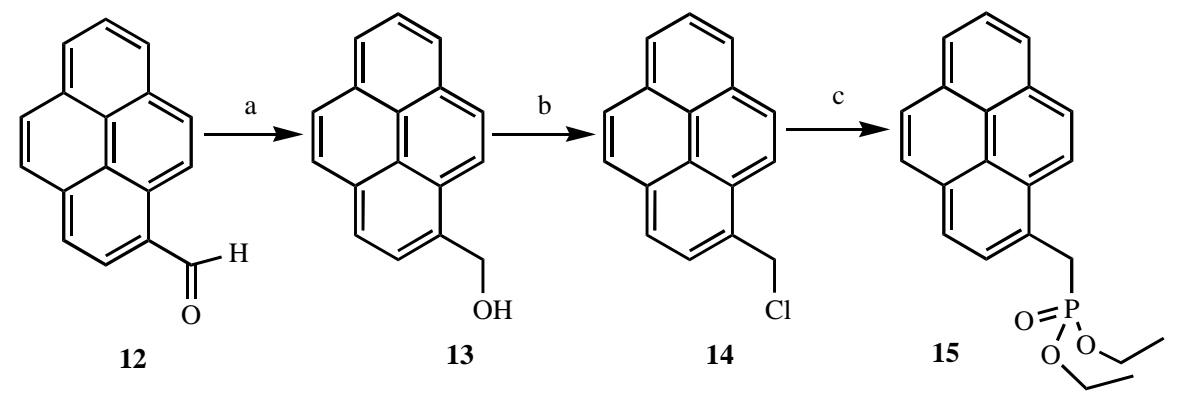

Scheme 6. Synthesis of the pyrene derivatives a) $\mathrm{LiAlH}_{4}$, THF, $0^{\circ} \mathrm{C}, \mathrm{N}_{2}$; b) $\mathrm{SOCl}_{2}, \mathrm{Py}, \mathrm{CH}_{2} \mathrm{Cl}_{2}$; c) $\mathrm{P}\left(\mathrm{OC}_{2} \mathrm{H}_{5}\right)_{3}$, tert-BuOK, THF.

The following signals were observed in the ${ }^{1} \mathrm{H}$ NMR spectrum of compound 13: one singlet at $\delta_{\mathrm{H}} 1.95$ for the $\mathrm{OH}$ proton, one singlet at $\delta_{\mathrm{H}} 5.31$ due to the $\mathrm{CH}_{2}$ group and for the pyrene group (multiplets at $\delta_{\mathrm{H}}$ 7.93- 8.29). The formation of the 1-(chloromethyl)pyrene 14 and the diethyl pyren1-ylmethylphosphonate $\mathbf{1 5}$ was confirmed by X-ray diffraction studies as shown in Figs. (3 and 4).
To obtain the donor-bridge system of first and second generation, we used the same methodology reported above between the dialdehyde $\mathbf{3}$, or $\mathbf{1 1}$ and the diethyl pyren-1ylmethylphosphonate 15 (Scheme 7).

In the ${ }^{1} \mathrm{H}$ NMR spectrum of the first generation aldehyde 16 two doublets were observed at $\delta_{\mathrm{H}} 7.57$ and at $\delta_{\mathrm{H}} 7.72$ due 

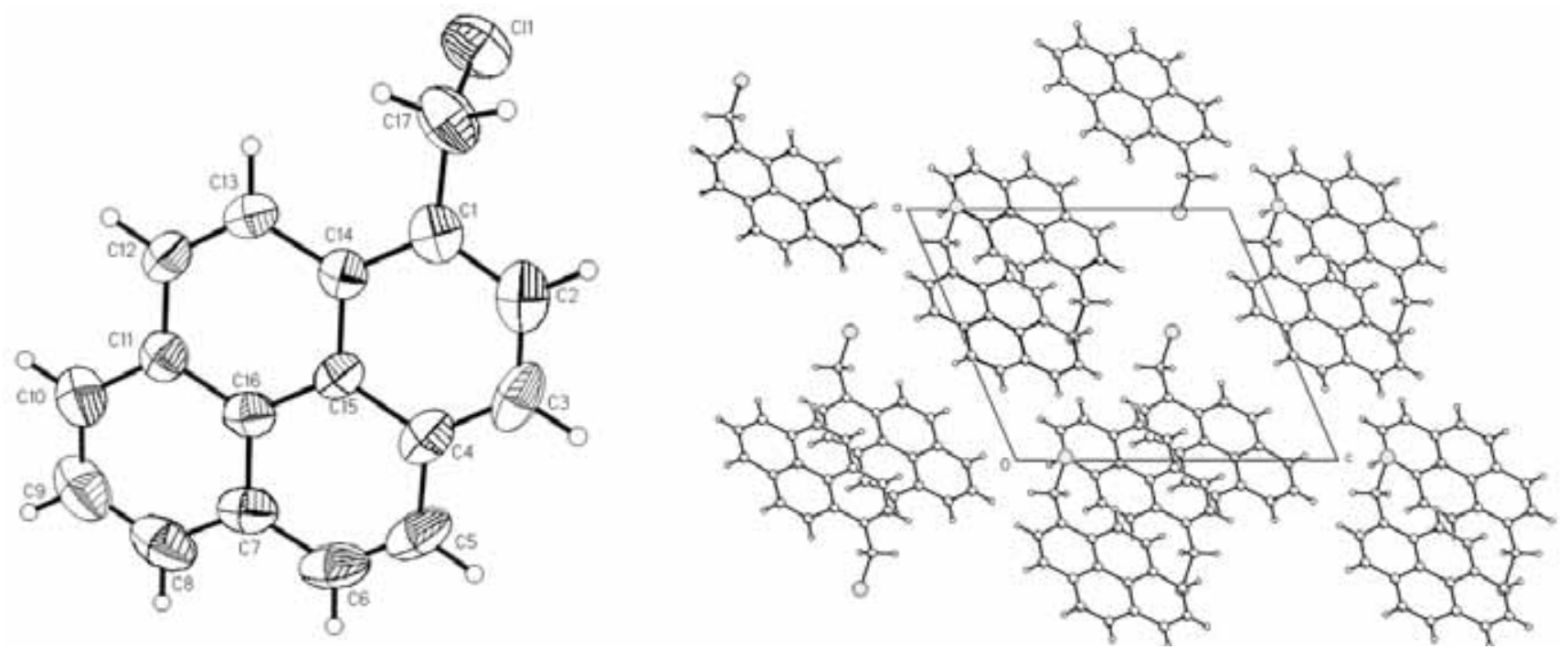

Fig. (3). Crystal structure and crystal packing of the 1-(chloromethyl)pyrene 14. Selected bond lengths $[\AA]$ : $\mathrm{Cl}(1)-\mathrm{C}(17)=1.801$, $\mathrm{C}(1)$ $\mathrm{C}(17)=1.491$. Selected angles $\left({ }^{\circ}\right)$ : $\mathrm{C}(2)-\mathrm{C}(1)-\mathrm{C}(17)=118.5, \mathrm{C}(14)-\mathrm{C}(1)-\mathrm{C}(17)=122.1, \mathrm{C}(1)-\mathrm{C}(17)-\mathrm{Cl}(1)=110.88$.
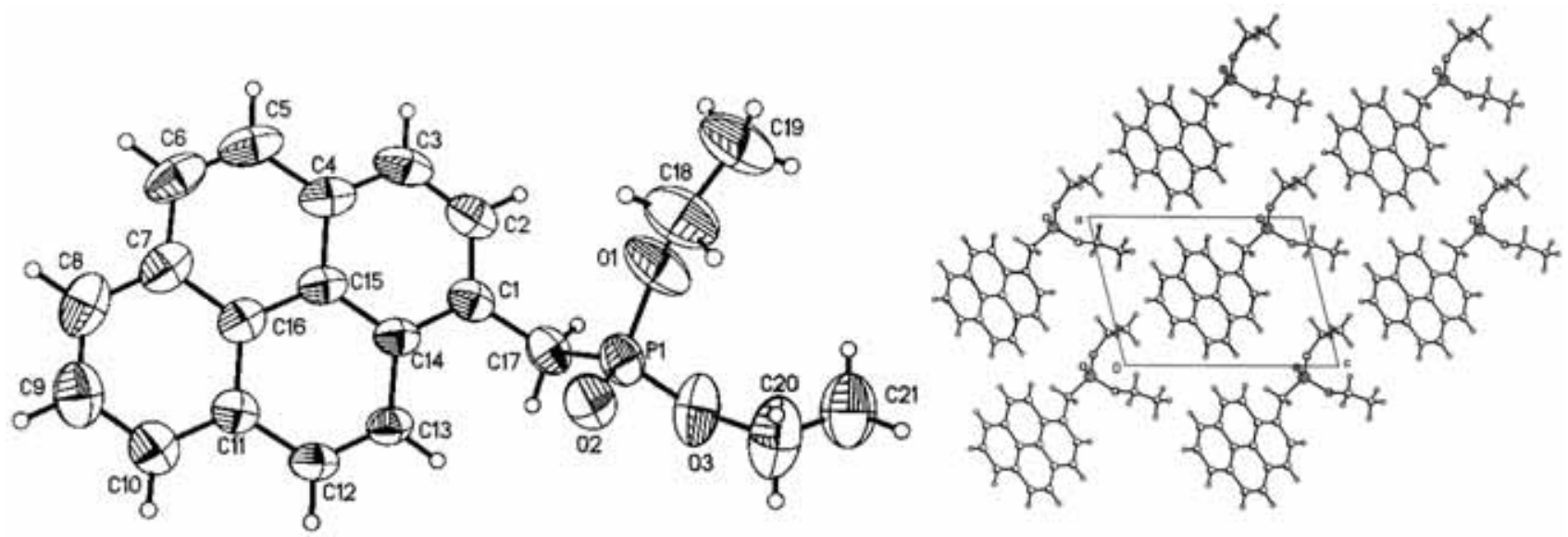

Fig. (4). Crystal structure and crystal packing of diethyl pyren-1-ylmethylphosphonate 15. Selected bond lengths $[\AA]$ : $\mathrm{Cl}(1)-\mathrm{C}(17)=1.801$, $\mathrm{C}(1)-\mathrm{C}(17)=1.491$. Selected angles $\left({ }^{\circ}\right)$ : $\mathrm{O}(2)-\mathrm{P}(1)-\mathrm{O}(1)=115.16, \mathrm{O}(2)-\mathrm{P}(1)-\mathrm{O}(3)=114.04, \mathrm{O}(1)-\mathrm{P}(1)-\mathrm{O}(3)=104.98, \mathrm{O}(2)-\mathrm{P}(1)-\mathrm{C}(17)=117.05$, $\mathrm{O}(1)-\mathrm{P}(1)-\mathrm{O}(17)=103.20, \mathrm{C}(1)-\mathrm{C}(17)-\mathrm{P}(1)=113.34, \mathrm{C}(14)-\mathrm{C}(1)-\mathrm{C}(17)=122.0$.

to the vinylic protons with coupling constants $J=15.0$ and $J$ $=14.7 \mathrm{~Hz}$, respectively. Also the characteristic signals due to the pyrene moiety were observed at $\delta_{\mathrm{H}} 7.58$ to 8.05 , and finally one singlet at $\delta_{\mathrm{H}} 11.58$ assigned to the aldehyde proton.

The ${ }^{1} \mathrm{H}$ NMR spectrum of compound 17 showed one triplet at $\delta_{\mathrm{H}} 0.95$ due to the methyl groups, two doublets at $\delta_{\mathrm{H}}$ 1.53 and 1.83 assigned to the $\mathrm{CH}_{2}$ groups of the aliphatic chain, one triplet at $\delta_{\mathrm{H}} 4.17$ due to the $\mathrm{CH}_{2}-\mathrm{O}$ groups, six doublets at $\delta_{\mathrm{H}} 7.25-8.44$ due to the vinylic protons, with coupling constants $J=16.2 \mathrm{~Hz}$, multiplets due to the anthracene and pyrene groups, and one singlet at $\delta_{\mathrm{H}} 11.55$ assigned to the proton of the aldehyde group. The structures of the aldehydes 16 and 17 were confirmed by FAB+ mass spectrometry.

Compound 11, pyrene carboxaldehyde $\mathbf{1 2}$ and the anthracene dialdehyde 2 were used to obtain pyrene derivative 19 using the same Horner-Wadsworth-Emmons reaction upon the conditions described above (Scheme 8).

In the ${ }^{1} \mathrm{H}$ NMR spectrum of the compound 18 one doublet was observed at $\delta 3.33$ with a coupling constant of $J=$
$21.9 \mathrm{~Hz}$, due to the methylene group joined to the phosphonate fragment $\left(\mathrm{CH}_{2}-\mathrm{P}\right)$. In addition, signals assigned to the anthracenyl group were observed: one singlet at $\delta 8.86$, one doublet at $\delta 8.41$ with a coupling constant $J=6.9 \mathrm{~Hz}$ double of doublets at $\delta 8.1$ with coupling constants $J=6.4 \mathrm{~Hz}$, and finally one multiplet at $\delta 7.4$. In the ${ }^{13} \mathrm{C}$ NMR spectrum of compound 19 characteristic signals for the anthracene and the pyrene moietys were observed at $\delta 111.3-134.8$.

Fig. (5) shows the UV-Vis spectra of $\mathrm{CH}_{2} \mathrm{Cl}_{2}$ solutions of the dialdehydes 2, 7 (Fig. 5a) and the corresponding pyrene containing systems 16, 17 and 19 (Fig. 5b). The absorption spectra of dialdehydes 2,7 showed two main peaks at 232237 and 264-270 $\mathrm{nm}$ and broad band with maximum at 423 $\mathrm{nm}$. The presence of one more anthracene and the styryl group in compounds 16, 17 and 19 provoked an ipsochromic shift in the firs two $t$ peaks, and the broad signal became bigger and its maximum was shifted to $427 \mathrm{~nm}$. In the case of the pyrene-monoaldehyde 16, the signals became broader and the most significant change is the appearance of two maxima observed at 334 and at $363 \mathrm{~nm}$, attributed to the pyrene moiety. These maxima were red shifted passing from 

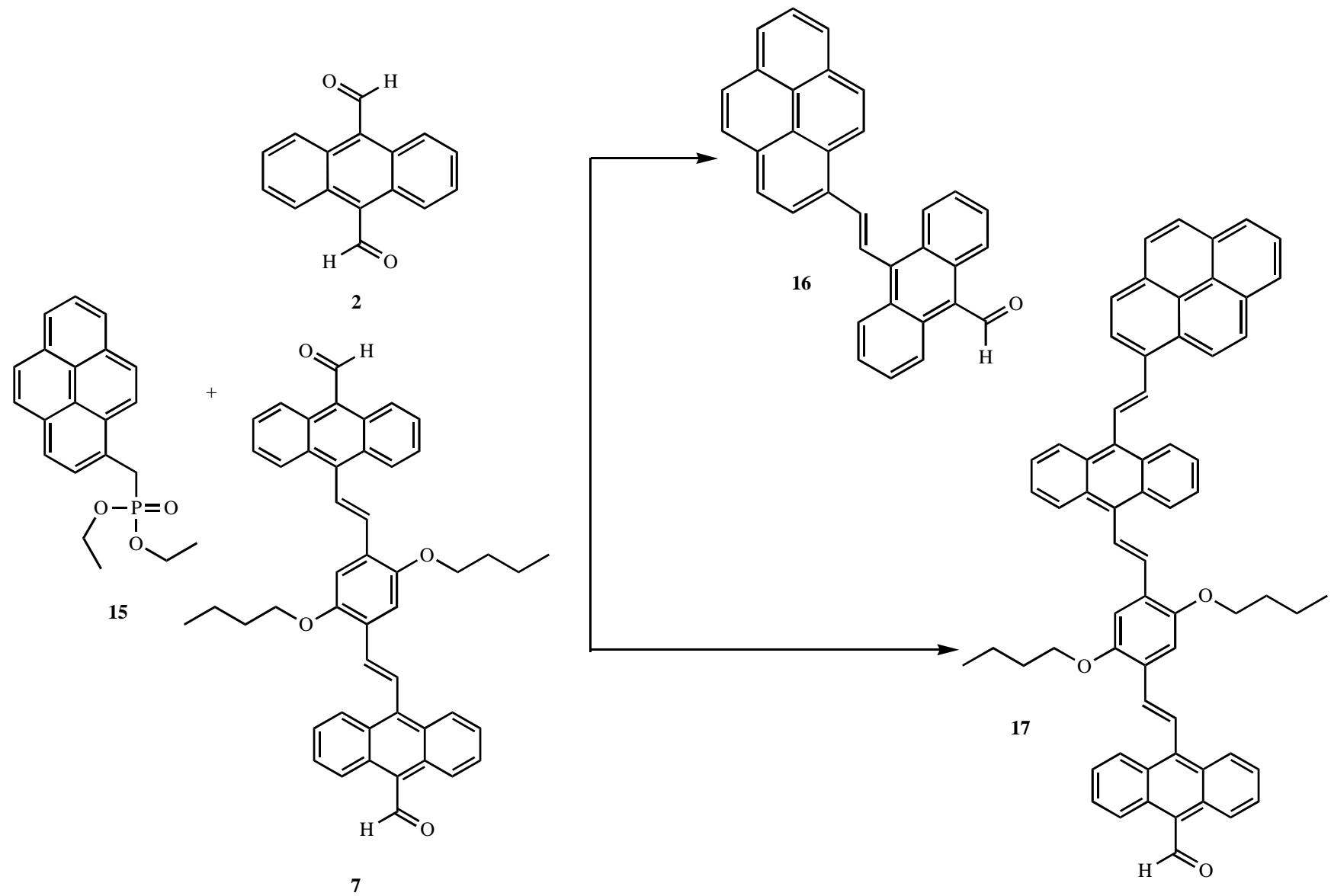

Scheme 7. Synthesis of the rods of first $\mathbf{1 6}$ and second $\mathbf{1 7}$ generation (tert-BuOK in dry THF).

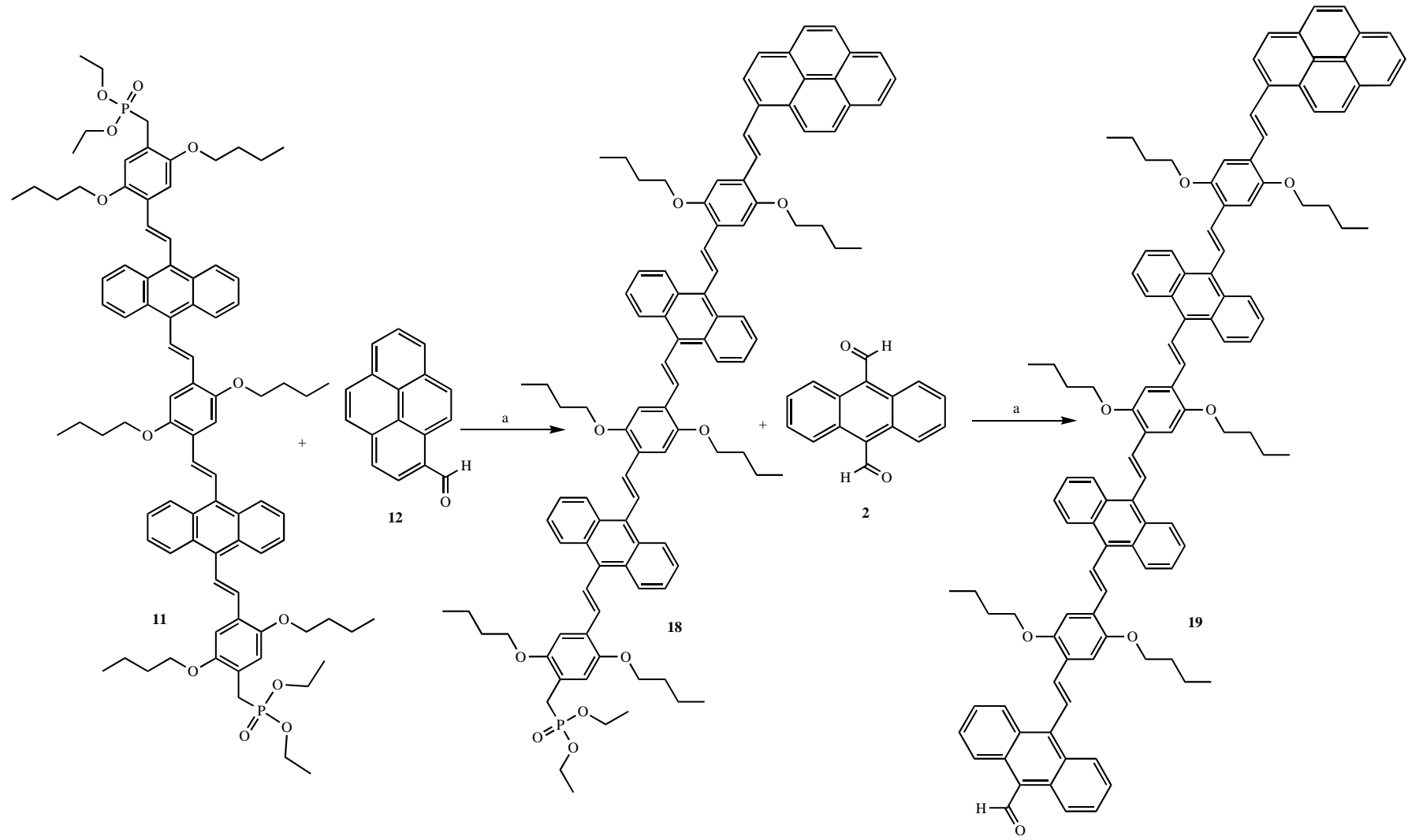

Scheme 8. Synthesis of the molecular antenna of third generation $\mathbf{1 9}$ a) tert-BuOK, dry THF. 

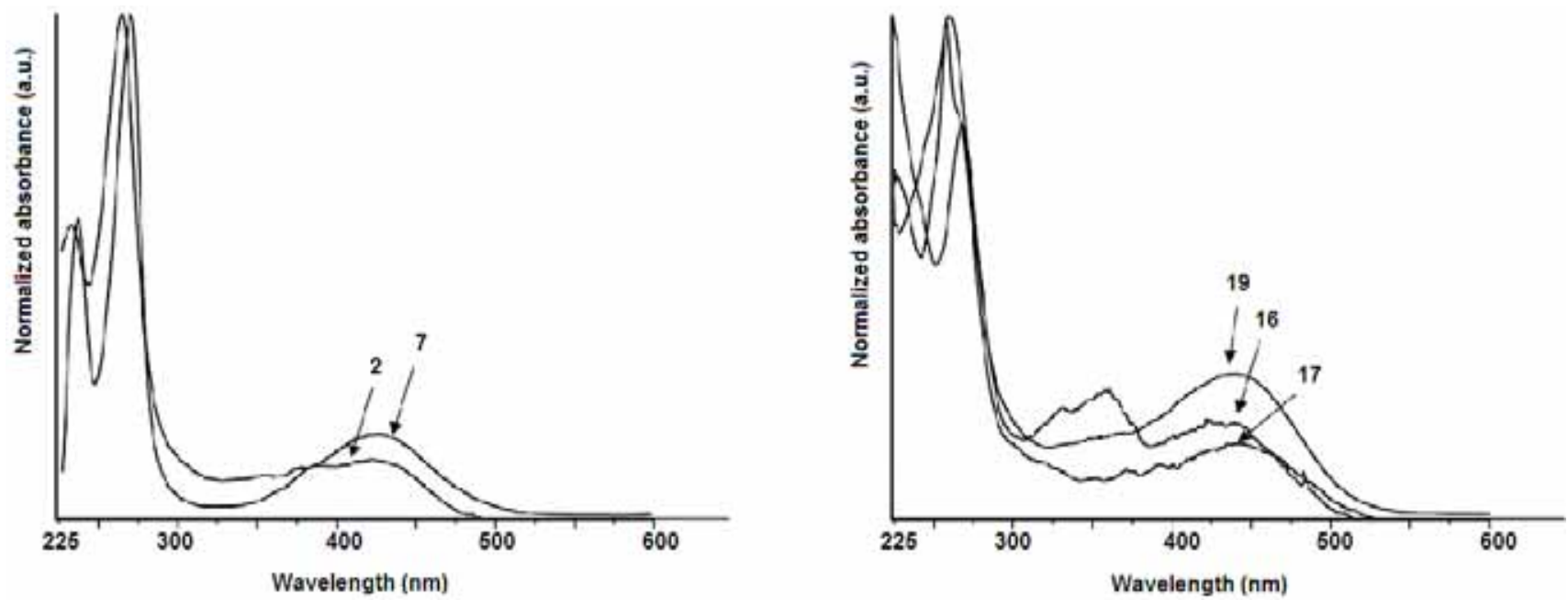

Fig. (5). UV-vis spectra of $\mathrm{CH}_{2} \mathrm{Cl}_{2}$ solutions of a) dialdehydes 2 and 7; b) monoaldehydes 16,17 and 19.

Table 1. UV-vis Data of the Dialdehydes 2, 7 and Monoaldehydes 16, 17 and 19

\begin{tabular}{|c|c|c|}
\hline Compound & $\boldsymbol{\lambda}_{\max }(\mathbf{n m})$ & Band gap, eV \\
\hline \hline $\mathbf{2}$ & $237,270,423$ & 4.30 \\
\hline $\mathbf{7}$ & $232,264,427$ & 4.13 \\
\hline $\mathbf{1 6}$ & $228,270,334,363,443$ & 4.13 \\
\hline $\mathbf{1 7}$ & $230,261,273,374,394,445$ & 4.12 \\
\hline $\mathbf{1 9}$ & $224,260,348,449$ & 4.11 \\
\hline
\end{tabular}

the first $\mathbf{1 6}$ to the second generation $\mathbf{1 7}$ which is in agreement with the higher conjugation of the dianthracenyl in comparison with the mono anthracenyl fragment. In the case of compound $\mathbf{1 7}$ the band attributed to the anthracene moiety was strongly diminished. Sharpness in the peaks at 400 to $500 \mathrm{~nm}$ is observed suggesting a higher molecular order in solution for the greater molecules. The fact that the optical absorption properties of the pyrene-anthracene and pyreneanthracene-styrene-anthracene fragments are not affected by the nature of the aliphatic chain in the styrene are coincident with those of the corresponding conjugated model molecules suggests that each moiety in the rod acts as an isolated molecule.

\section{CONCLUSIONS}

Fully conjugated rods with anthrylene vinylene and phenylene vinylene repeated units were successfully synthesized by Horner-Wadsworth-Emmons reaction utilizing potassium tert-butoxide in dry THF. The synthesized rods which have butylene groups as solubility spacers in the main chain exhibited good solubility in polar solvents. The solutions of the synthesized pyrene- containing molecular rods exhibited a blue shift in the UV-vis from the absorption maximum due to presence of the pyrene group compared to the dialdehydes.

\section{EXPERIMENTAL SECTION}

\section{Materials and Equipments}

Solvents and reagents were purchased as reagent grade and used without further purification. Acetone was distilled over calcium chloride. Tetrahydrofuran was distilled from sodium and benzophenone. Column chromatography was performed on Merck silica gel $60 \AA$ (70-230 mesh). ${ }^{1} \mathrm{H}$ and ${ }^{13} \mathrm{C}$ NMR spectra were recorded on a Varian-Unity-300 MHz with tetra-methylsilane (TMS) as an internal reference. Infrared (IR) spectra were measured on a Nicolet FT-SSX spectrophotometer. Elemental analyses were performed by Galbraith Laboratories, INC Knoxville. FAB+ mass spectra were taken on a JEOL JMS AX505 HA instrument. Electrospray mass spectra were taken on a Bruker Daltonic, Esquire 6000.

\section{9,10-Bis(chloromethyl)anthracene (1)}

1,4-dixane $40 \mathrm{ml}$ of and hydrochloric acid $5 \mathrm{ml}$ were saturated with hydrogen chloride gas, prepared form sodium chloride and sulfuric acid. Then anthracene $5 \mathrm{~g}(28.08 \mathrm{mmol})$ was added. The mixture was stirred slowly and heated to $60^{\circ} \mathrm{C}$ overnight. The fine granular solid was filtered and dissolved in dichloromethane and washed with water. The solvent was removed in vacuum, and the residue was chromatographed on alumina (hexane-diethyl ether, 3:1) to give 9,10-bis(chloromethyl)anthracene (1) as a yellow powder, yield $4.8 \mathrm{~g}, 17.51 \mathrm{mmol}(63 \%)$, m.p. $280-282{ }^{\circ} \mathrm{C}$ (lit [15] m.p. 253-255). FTIR (pellet, $\mathrm{KBr}, \mathrm{cm}^{-1}$ ): 3057, 1952, 1706, $1621,1525,1475,1444,1245,1181,1032,764,689,620$, 559. 405, 383, 364, 263, 232. ${ }^{1} \mathrm{H}$ NMR (200 MHz, DMSOd6), $\delta$ (ppm): 5.86 (s, 4H, $\left.\mathrm{CH}_{2}-\mathrm{Cl}\right), 7.69$ (m, 4H, Ar-H), 8.49 (m, 4H, Ar-H). ${ }^{13} \mathrm{C}$ NMR (DMSO-d6), $\delta(\mathrm{ppm}): 124.7$ (Ar), 126.7 (Ar), 129.2 (Ar), 130.6 (Ar), $132.6\left(\mathrm{C}_{\text {ipso }}\right) . \mathrm{EM}: \mathrm{m} / \mathrm{z}$ : $274[\mathrm{M}]^{+}$Calc. for $\mathrm{C}_{16} \mathrm{H}_{12} \mathrm{Cl}_{2}$ : C, 69.84; H, 4.40 (\%). Found: $\mathrm{C}, 69.83 ; \mathrm{H}, 4.38$. 


\section{Anthracene-9,10-dicarbaldehyde (2)}

A stirred suspension of 9,10-bis(chloromethyl)anthracene (1) $1 \mathrm{~g}(3.6 \mathrm{mmol})$ and anhydrous dimethyl sulfoxide $20 \mathrm{ml}$ under nitrogen at room temperature were added slowly to a solution of sodium $0.2 \mathrm{~g}(8.6 \mathrm{mmol})$, ethanol $12 \mathrm{ml}$ and 2nitropropane $1.2 \mathrm{ml}(13.6 \mathrm{mmol})$. The mixture was stirred for $12 \mathrm{~h}$. Cold water was added, and the precipitate was filtered and dried. The product was purified by silica gel chromatography using hexane as an eluent to give 9,10anthracenedicarboxaldehyde (2) as an orange powder, yield 0.7 g, 2.99 mmol (83\%), m.p. 243-244 ${ }^{\circ} \mathrm{C}$. FTIR (pellet, $\left.\mathrm{KBr}, \mathrm{cm}^{-1}\right): 3080,2860,1677,1444,1353,1177,1022,889$, 744, 562. UV-Vis $\left(\mathrm{CH}_{2} \mathrm{Cl}_{2}, \mathrm{~nm}\right) \lambda_{\max }: 437,265 .{ }^{1} \mathrm{H}$ NMR (DMSO-d6, $200 \mathrm{MHz}), \delta(\mathrm{ppm}) 7.67$ (m, 4H, Ar-H), 8.69 (m, 4H, Ar-H), 11.44 (s, 2H, HC=O). ${ }^{13} \mathrm{C}$ NMR (DMSO-d6), $\delta(\mathrm{ppm}): 124.1$ (Ar), 128.3 (Ar), 130.2 (Ar), $131.6\left(\mathrm{C}_{\mathrm{ipso}}\right)$, $194.2(\mathrm{HC}=\mathrm{O})$. EM: m/z: $234[\mathrm{M}]^{+}$Calc. for $\mathrm{C}_{16} \mathrm{H}_{10} \mathrm{O}_{2}: \mathrm{C}$, 82.04; H, 4.30 (\%). Found: C, 82.03; H, 4.31 (\%).

\section{0-Vinylanthracene-9-carboxaldehyde (3)}

9,10- anthracenedicarboxaldehyde (2) $2 \mathrm{~g}$ (8.53 mmol) was added to the suspension of methyltriphenilphosphonium bromide $3.04 \mathrm{~g}(8.53 \mathrm{mmol})$ and $n$-Buthyl lithium $3.28 \mathrm{ml}$ $(8.52 \mathrm{mmol})$ under nitrogen at $0^{\circ} \mathrm{C}$ in dry THF $40 \mathrm{ml}$. The solution was stirred overnight. After that, a few drops of water were added and the mixture was concentrated and purified by silica gel chromatography using a mixture of hexanedichloromethane 90:10 as an eluent to give 10vinylanthracene-9-carboxaldehyde (3) as orange crystals, yield $1.5 \mathrm{~g}, 6.48 \mathrm{mmol}$ (76\%), m.p. 93-95 ${ }^{\circ} \mathrm{C}$. FTIR. (pellet, $\mathrm{KBr}, \mathrm{cm}^{-1}$ ): 3056, 2927, 1675, 1437, 1188, 1118, 721, 542. $\mathrm{UV}$-vis $\left(\mathrm{CHCl}_{3}, \mathrm{~nm}\right) \lambda_{\max }: 403,382,265 .{ }^{1} \mathrm{H} \mathrm{NMR}(300$ $\left.\mathrm{MHz}, \mathrm{CDCl}_{3}\right), \delta_{\mathrm{H}}(\mathrm{ppm}): 5.48\left(\mathrm{dd}, 1 \mathrm{H},=\mathrm{CH}_{2}, J_{\text {trans }}=18 \mathrm{~Hz}\right)$, $5.97\left(\mathrm{dd}, 1 \mathrm{H},=\mathrm{CH}_{2}, J_{\text {cis }}=11.7 \mathrm{~Hz}\right), 7.25\left(\mathrm{q}, 1 \mathrm{H},=\mathrm{CH}, J_{\text {trans }}=\right.$ $\left.18 \mathrm{~Hz}, J_{\text {cis }}=11.7 \mathrm{~Hz}\right), 7.40(\mathrm{~m}, 2 \mathrm{H}, \mathrm{Ar}-\mathrm{H}), 7.53(\mathrm{~m}, 2 \mathrm{H}, \mathrm{Ar}-$ $\mathrm{H}), 8.22(\mathrm{~m}, 2 \mathrm{H}, \mathrm{Ar}-\mathrm{H}), 8.80(\mathrm{~m}, 2 \mathrm{H}, \mathrm{Ar}-\mathrm{H}), 11.30(\mathrm{~s}, 1 \mathrm{H}$, $\mathrm{HC}=\mathrm{O}) .{ }^{13} \mathrm{C} \mathrm{NMR}\left(\mathrm{CDCl}_{3}\right), \delta(\mathrm{ppm}) 122.9\left(=\mathrm{CH}_{2}\right), 124.1$ $\left(\mathrm{C}_{\mathrm{ipso}}\right), 125.0(\mathrm{Ar}), 126.2(\mathrm{Ar}), 128.3(\mathrm{Ar}), 128.5(\mathrm{Ar}), 128.8$ $\left(\mathrm{C}_{\mathrm{ar}}\right), 133.1\left(\mathrm{C}_{\mathrm{ar}}\right), 133.3\left(\mathrm{C}_{\mathrm{ipso}}\right), 133.8(=\mathrm{CH}), 193.2(\mathrm{C}=\mathrm{O})$. EM: m/z: $232[\mathrm{M}]^{+}$. Calc. for $\mathrm{C}_{17} \mathrm{H}_{12} \mathrm{O}: \mathrm{C}, 87.90 ; \mathrm{H}, 5.21$ (\%). Found: C, 87.92; H, $5.23(\%)$.

\section{9, 10-Divinylanthracene (4)}

Yield $0.19 \mathrm{~g}, 0.85 \mathrm{mmol}(10 \%)$, yellow crystals, m.p 84$86{ }^{\circ} \mathrm{C}$. FTIR. (pellet, $\mathrm{KBr}, \mathrm{cm}^{-1}$ ): 3056, 2927, 1675, 1437, 1188, 1118, 721, 542. UV-vis $\left(\mathrm{CHCl}_{3}, \mathrm{~nm}\right) \lambda_{\max }: 403,382$, 265. ${ }^{1} \mathrm{H}$ NMR $\left(200 \mathrm{MHz}, \mathrm{CDCl}_{3}\right), \delta(\mathrm{ppm}): 5.45(\mathrm{dd}, 1 \mathrm{H}$, $\left.=\mathrm{CH}_{2}, J_{\text {trans }}=17.8 \mathrm{~Hz}\right), 5.98\left(\mathrm{dd}, 1 \mathrm{H},=\mathrm{CH}_{2}, J_{\text {cis }}=11.4 \mathrm{~Hz}\right)$, $7.38-7.53\left(\mathrm{~m}, 6 \mathrm{H}, \mathrm{H}-\mathrm{Ar},=\mathrm{CH}, J_{\text {trans }}=16.4 \mathrm{~Hz}, J_{\text {cis }}=10.2 \mathrm{~Hz}\right)$, $8.33\left(\mathrm{dd}, 4 \mathrm{H}, \mathrm{H}-\mathrm{Ar}, J_{\text {cis }}=11.7 \mathrm{~Hz}\right) .{ }^{13} \mathrm{C} \mathrm{NMR}\left(\mathrm{CDCl}_{3}\right)$, $\delta$ (ppm): $123.0\left(=\mathrm{CH}_{2}\right), 125.8$ (Ar), 126.3 (Ar), 128.9 (Car), $133.5\left(\mathrm{C}_{\mathrm{ipso}}\right), 133.8(=\mathrm{CH})$. ). EM: m/z: $230[\mathrm{M}]^{+}$. Calc. for $\mathrm{C}_{18} \mathrm{H}_{14}$ : C, 93.87; H, 6.13 (\%). Found: C, 93.85; H, $6.15(\%)$.

\section{1,4-Dibutoxybenzene (5)}

Ground $\mathrm{NaOH}(16.0 \mathrm{~g}, 285 \mathrm{mmol})$ was added to $\mathrm{EtOH}$ $(250 \mathrm{~mL})$, and the mixture was stirred for $20 \mathrm{~min}$. Upon addition of hydroquinone (12.1 g, $110 \mathrm{mmol})$, the solution turned dark brown, it was stirred for another $30 \mathrm{~min}$, and heated to reflux. After $15 \mathrm{~min}$, (S)-2- methylbutyl- $p$ toluenesulfonate $(58.64 \mathrm{~g}, 242 \mathrm{mmol})$ was added dropwise. The resulting suspension was stirred for another $16 \mathrm{~h}$, cooled to room temperature, and filtered. The solvent was evaporated, and the residue was purified by silica gel chromatography using hexane as an eluent to afford 1,4dibutoxybenzene (5) as white flakes, yield $9.52 \mathrm{~g}, 42.8 \mathrm{mmol}$ (94 \%) m.p. $45-47{ }^{\circ} \mathrm{C}$ (lit. [14] m.p. 44.5-45.2). FTIR. (pellet, $\mathrm{KBr}, \mathrm{cm}^{-1}$ ): 2958, 2935, 1866, 1510, 1471, 1235, 1121, $1042,828,760$. UV-vis $\left(\mathrm{CHCl}_{3}, \mathrm{~nm}\right) \lambda_{\max }: 293,242 .{ }^{1} \mathrm{H}$ NMR $\left(200 \mathrm{MHz}, \mathrm{CDCl}_{3}\right), \delta(\mathrm{ppm}): 0.96\left(\mathrm{t}, 6 \mathrm{H}, \mathrm{CH}_{3}, J=6.9\right.$ $\mathrm{Hz}), 1.47\left(\mathrm{~m}, 4 \mathrm{H}, \mathrm{CH}_{2}\right), 1.73\left(\mathrm{~m}, 4 \mathrm{H}, \mathrm{CH}_{2}\right), 3.90\left(\mathrm{t}, 4 \mathrm{H}, \mathrm{CH}_{2}\right.$, $J=6.7 \mathrm{~Hz}), 6.83(\mathrm{~s}, 2 \mathrm{H}, \mathrm{Ar}-\mathrm{H}) .{ }^{13} \mathrm{C} \mathrm{NMR}\left(\mathrm{CDCl}_{3}\right), \delta(\mathrm{ppm})$ : $13.8\left(\mathrm{CH}_{3}\right), 19.2\left(\mathrm{CH}_{2}\right), 31.4\left(\mathrm{CH}_{2}\right), 68.3\left(\mathrm{CH}_{2}-\mathrm{O}\right), 115.3$ (Ar), 153.1 (Ar-O). EM: m/z: $222[\mathrm{M}]^{+}$. Calc. for $\mathrm{C}_{14} \mathrm{H}_{22} \mathrm{O}_{2}$ : C, 75.63; H, 9.97; Found: C, 75.65; H, 9.96 (\%).

\section{1,4-Dibromo-2,5-dibutoxybenzene (6)}

To a solution of 5 (9.04 g, $20.24 \mathrm{mmol})$ in carbon tetrachloride $(100 \mathrm{~mL})$, were added to $2.6 \mathrm{~mL}$ of bromine. The reaction mixture was heated to $60{ }^{\circ} \mathrm{C}$ and stirred for $48 \mathrm{~h}$. Then a solution $1 \mathrm{~N}$ of $\mathrm{NaOH}$ was added to the reaction mixture. The organic phase was partially evaporated under vacuum and the residue was precipitated from hexane, to obtain 1,4-dibromo-2,5-dibutoxybenzene (6), as a white solid, yield 12.8 g, 33.86 mmol (90 \%), m.p. 76-77 ${ }^{\circ} \mathrm{C}$ (lit. [16] m.p. 72 ${ }^{\circ} \mathrm{C}$ ) FTIR (pellet, KBr, cm-1): 2959, 2934, 2871, 1506, 1470, 1229, 1032, 824. UV-vis $\left(\mathrm{CHCl}_{3}, \mathrm{~nm}\right) \lambda_{\max }: 301,242 .{ }^{1} \mathrm{H}$ NMR (200 MHz, $\left.\mathrm{CDCl}_{3}\right), \delta(\mathrm{ppm}): 0.97\left(\mathrm{t}, 6 \mathrm{H}, \mathrm{CH}_{3}, J=6.6\right.$ $\mathrm{Hz}), 1.53\left(\mathrm{~m}, 4 \mathrm{H}, \mathrm{CH}_{2}\right), 1.78\left(\mathrm{~m}, 4 \mathrm{H}, \mathrm{CH}_{2}\right), 3.98\left(\mathrm{t}, 4 \mathrm{H}, \mathrm{CH}_{2}\right.$, $J=6.4 \mathrm{~Hz}), 4.63\left(\mathrm{~s}, 4 \mathrm{H}, \mathrm{CH}_{2}-\mathrm{Cl}\right), 6.91(\mathrm{~s}, 2 \mathrm{H}, \mathrm{Ar}-\mathrm{H}) .{ }^{13} \mathrm{C}$ NMR $\left(\mathrm{CDCl}_{3}\right), \delta(\mathrm{ppm}): 13.8\left(\mathrm{CH}_{3}\right), 19.2\left(\mathrm{CH}_{2}\right), 31.4\left(\mathrm{CH}_{2}\right)$, $41.6\left(\mathrm{CH}_{2}-\mathrm{Cl}\right), 68.2\left(\mathrm{CH}_{2}-\mathrm{O}\right), 115.2(\mathrm{Ar}), 150.8$ (Ar-O), 153.0 (Ar-O). EM: m/z: $318[\mathrm{M}]^{+}$. Calc. for $\mathrm{C}_{14} \mathrm{H}_{20} \mathrm{O}_{2} \mathrm{Br}_{2}: \mathrm{C}$, 60.19; H, 7.58; Found C, 60.18; H, 7.57 (\%).

\section{1,4-Dibutoxy-2,5-bis(chloromethyl)benzene (9)}

A solution of 5 (10.04 g, $40.1 \mathrm{mmol})$ in 1,4-dioxane (60 $\mathrm{mL}$ ) was cooled to $0{ }^{\circ} \mathrm{C}$, and aqueous $\mathrm{HCl}(35 \mathrm{~mL}) 37 \%$ was added. $\mathrm{HCl}$ gas was bubbled through the solution while $36 \%$ aqueous formaldehyde $(20 \mathrm{~mL})$ was added dropwise, the mixture was stirred at room temperature for $1.5 \mathrm{~h}$ and then it was heated to $60{ }^{\circ} \mathrm{C}$ and stirred for $16 \mathrm{~h}$. The mixture was partially evaporated and methanol $(150 \mathrm{~mL})$ was added, the suspension was filtered. The solid was recrystallized from hexane to afford 1,4-dibutoxy-2,5-dichloromethylbencene (9) as a white powder, yield $12.8 \mathrm{~g}, 40.23 \mathrm{mmol}$ (90.1 \%), m.p 86-87 ${ }^{\circ}$ C. FTIR. (pellet, $\mathrm{KBr}, \mathrm{cm}^{-1}$ ): 2959, 2934, 2871, $1506,1470,1229,1032,824$. UV-vis $\left(\mathrm{CHCl}_{3}, \mathrm{~nm}\right) \lambda_{\max }: 301$, 242. ${ }^{1} \mathrm{H} \mathrm{NMR}\left(200 \mathrm{MHz}, \mathrm{CDCl}_{3}\right), \delta(\mathrm{ppm}): 0.97$ (t, $6 \mathrm{H}, \mathrm{CH}_{3}$, $J=6.7 \mathrm{~Hz}), 1.53\left(\mathrm{~m}, 4 \mathrm{H}, \mathrm{CH}_{2}\right), 1.78\left(\mathrm{~m}, 4 \mathrm{H}, \mathrm{CH}_{2}\right), 3.98(\mathrm{t}$, $\left.4 \mathrm{H}, \mathrm{CH}_{2}, J=6.4 \mathrm{~Hz}\right), 4.63\left(\mathrm{~s}, 4 \mathrm{H}, \mathrm{CH}_{2}-\mathrm{Cl}\right), 6.91$ (s, 2H, Ar$\mathrm{H}) .{ }^{13} \mathrm{C} \mathrm{NMR}\left(\mathrm{CDCl}_{3}\right), \delta(\mathrm{ppm}): 13.8\left(\mathrm{CH}_{3}\right), 19.2\left(\mathrm{CH}_{2}\right)$, $31.4\left(\mathrm{CH}_{2}\right), 41.6\left(\mathrm{CH}_{2}-\mathrm{Cl}\right), 68.2\left(\mathrm{CH}_{2}-\mathrm{O}\right), 115.2(\mathrm{Ar}), 150.8$ (Ar-O), 153.0 (Ar-O). EM: m/z: $318[\mathrm{M}]^{+}$. Calc. for $\mathrm{C}_{16} \mathrm{H}_{24}$ $\mathrm{O}_{2} \mathrm{Cl}_{2}$ : C, 60.19; H, 7.58; Cl, 22.21 (\%); Found: C, 60.18; H, $7.56(\%)$.

\section{Tetraethyl (2,5-dibutoxy-1,4-phenylene)bis(methylene)dip- hosphonate (10)}

Triethyl phosphite $(8.1 \mathrm{ml}, 47.23 \mathrm{mmol})$ and $9(6.7 \mathrm{~g}$, $20.98 \mathrm{mmol}$ ) were stirred at $160{ }^{\circ} \mathrm{C}$ for $4 \mathrm{~h}$. The reaction mixture was cooled at room temperature and purified by silica gel chromatography using ethyl acetate as an eluent to afford 2,5-bis(buthyloxy)-1,4-bis(benzyl)phosphonate (10) 
as a yellow pale oil, yield $0.3 \mathrm{~g}, 19.20 \mathrm{mmol}$ (94.4\%), FTIR. (pellet, KBr, cm-1): 2960, 2934, 2872, 1507, 1217, 1030, 965, 754. UV-vis $\left(\mathrm{CHCl}_{3}, \mathrm{~nm}\right) \lambda \max : 296,241.1 \mathrm{H} \mathrm{NMR}$ (200 MHz, $\left.\mathrm{CDCl}_{3}\right), \delta(\mathrm{ppm}): 0.95\left(\mathrm{~m}, 6 \mathrm{H}, \mathrm{CH}_{3}\right), 1.24(\mathrm{~m}$, $\left.12 \mathrm{H}, \mathrm{CH}_{3}\right), 1.48\left(\mathrm{~m}, 4 \mathrm{H}, \mathrm{CH}_{2}\right), 1.73\left(\mathrm{~m}, 4 \mathrm{H}, \mathrm{CH}_{2}\right), 3.19$ (s, $2 \mathrm{H}, \mathrm{CH}_{2}-\mathrm{P}$ ), 3.26 (s, 2H, $\left.\mathrm{CH}_{2}-\mathrm{P}\right), 3.88$ (m, 4H, $\left.\mathrm{CH}_{2}-\mathrm{O}\right), 4.01$ (m, 8H, $\left.\mathrm{CH}_{2}-\mathrm{O}\right), 6.79$ (s, 2H, Ar-H). 13C NMR $\left(\mathrm{CDCl}_{3}\right), \delta$ (ppm): $13.5(\mathrm{CH} 3), 16.0\left(\mathrm{CH}_{3}\right), 18.9\left(\mathrm{CH}_{2}\right), 25.4\left(\mathrm{CH}_{2}\right), 27.3$ $\left(\mathrm{CH}_{2}\right), 31.2\left(\mathrm{CH}_{2}\right), 61.5\left(\mathrm{CH}_{2}-\mathrm{O}\right), 67.9\left(\mathrm{CH}_{2}-\mathrm{O}\right), 68.4\left(\mathrm{CH}_{2}-\right.$ O), 115.1 (Ar), $121.0\left(\mathrm{C}_{\mathrm{ipso}}\right), 150.2$ (Ar-O), 153.0 (Ar-O). EM: m/z: 522 [M]+. Calc. for $\mathrm{C}_{24} \mathrm{H}_{44} \mathrm{O}_{8} \mathrm{P}_{2}$ : C, 55.16; H, 8.49; P: 11.85 (\%). Found: C, 55.14; H, $8.50(\%)$;

\section{0,10'-(1E,1'E)-2,2'-(2,5-Dibutoxy-1,4-phenylene)bis(ethene- 2,1-diyl)dianthracene-9-carboxaldehyde (11)}

A solution of $\mathbf{1 0}(0.89 \mathrm{~g}, 1.7 \mathrm{mmol})$ and tert-BuOK $(0.47$ $\mathrm{g}, 4.18 \mathrm{mmol})$ in dry THF $(5 \mathrm{~mL})$, under nitrogen was added dropwise to aldehyde 2 (1 g, $4.26 \mathrm{mmol})$ dissolved in dry THF $(10 \mathrm{~mL})$. The reaction mixture was stirred for $5 \mathrm{~h}$. The solution was poured on crushed ice, and $6 \mathrm{M} \mathrm{HCl}(6 \mathrm{~mL})$ was added. The aqueous phase was extracted, and the organic layer was subsequently washed with an aqueous solution of $3 \mathrm{M} \mathrm{HCl}$. The organic layer was dried over $\mathrm{Na}_{2} \mathrm{SO}_{4}$; the solvent was removed in vacuum, and the residue was purified by aluminum oxide chromatography using hexane $/ \mathrm{CH}_{2} \mathrm{Cl}_{2} 2: 1$ as an eluent to afford $10,10^{\prime}-(1 \mathrm{E}, 1$ 'E)-2,2'(2,5-dibutoxy-1,4-phenylene)bis(ethene-2,1-diyl)dianthracene-9-carboxaldehyde (11) as an orange solid, yield $0.874 \mathrm{~g}$, $1.28 \mathrm{mmol}(30 \%)$, t, desc. $165^{\circ} \mathrm{C}$. FTIR. (pellet, $\left.\mathrm{KBr}, \mathrm{cm}^{-1}\right)$ : 2959, 2932, 1724, 1674, 1450, 1285, 1123, 1073, 736. UVvis $\left(\mathrm{CHCl}_{3}, \mathrm{~nm}\right) \lambda_{\text {max }}: 401,373,266,241 .{ }^{1} \mathrm{H}$ NMR (300 $\left.\mathrm{MHz}, \mathrm{CDCl}_{3}\right), \delta(\mathrm{ppm}): 0.93\left(\mathrm{t}, 6 \mathrm{H}, \mathrm{CH}_{3}, J=6.9 \mathrm{~Hz}\right), 1.55$ $\left(\mathrm{m}, 4 \mathrm{H}, \mathrm{CH}_{2}\right), 1.82\left(\mathrm{~m}, 4 \mathrm{H}, \mathrm{CH}_{2}\right), 4.16\left(\mathrm{t}, 4 \mathrm{H}, \mathrm{CH}_{2}-\mathrm{O}, J=6.6\right.$ $\mathrm{Hz}), 7.54\left(\mathrm{~m}, 8 \mathrm{H},=\mathrm{CH}, \mathrm{Ar}-\mathrm{H}, J_{\text {trans }}=16.8 \mathrm{~Hz}\right), 7.69(\mathrm{~m}, 6 \mathrm{H}$, $\left.=\mathrm{CH}, \mathrm{Ar}-\mathrm{H}, J_{\text {trans }}=17.1 \mathrm{~Hz}\right), 8.06(\mathrm{dd}, 4 \mathrm{H}, \mathrm{Ar}-\mathrm{H}, J=8.2,8.0$ $\mathrm{Hz}$, 8.98 (dd, 4H, Ar-H, J=8.0 Hz), 11.54 (s, 2H, HC=O). ${ }^{13} \mathrm{C} \mathrm{NMR}\left(\mathrm{CDCl}_{3}\right), \delta(\mathrm{ppm}): 13.6\left(\mathrm{CH}_{3}\right), 19.1\left(\mathrm{CH}_{2}\right), 30.5$ $\left(\mathrm{CH}_{2}\right), 65.5\left(\mathrm{CH}_{2}-\mathrm{O}\right), 123.5(=\mathrm{CH}), 125.6(=\mathrm{CH}), 126.8$ $\left(\mathrm{C}_{\mathrm{ipso}}\right), 127.4\left(\mathrm{C}_{\mathrm{ipso}}\right), 128.5(\mathrm{Ar}), 128.8\left(\mathrm{C}_{\mathrm{Ar}}\right), 129.1\left(\mathrm{C}_{\mathrm{Ar}}\right)$, 129.2 (Ar), 130.8 (=C), $192.9(\mathrm{HC}=\mathrm{O}) . \mathrm{FAB}^{+} \mathrm{Ms} ;(\mathrm{m} / \mathrm{z}): 682$ $[\mathrm{M}]^{+}$. Calc. for $\mathrm{C}_{48} \mathrm{H}_{42} \mathrm{O}_{4}$ : C, 84.43; H, 6.20 (\%). Found: C, 84.43; H, $6.22(\%)$.

Tetraethyl (4,4'-(1E,1'E)-2,2'-(10,10'-(1E,1'E)-2,2'-(2,5dibutoxy-1,4-phenylene)bis(ethene-2,1-diyl)bis (anthracene10,9-diyl)) bis(ethene-2,1-diyl) bis(2,5-dibutoxy-4,1phenylene))bis(methylene)diphosphonate (12) as a yellow solid, yield $0.57 \mathrm{~g}, 0.4 \mathrm{mmol}(24 \%)$, t, desc. 230oC. FTIR. (pellet, KBr, cm-1): 3043, 2956, 2931, 2868, 1499, 1384, 1203, 1029, 755. UV-vis $\left(\mathrm{CHCl}_{3}, \mathrm{~nm}\right) \lambda$ max: 447, 344, 265. 1H NMR (300 MHz, $\left.\mathrm{CDCl}_{3}\right), \delta(\mathrm{ppm}): 0.94\left(\mathrm{t}, 9 \mathrm{H}, \mathrm{CH}_{3}, \mathrm{~J}=\right.$ $6.6 \mathrm{~Hz}), 1.02\left(\mathrm{t}, 9 \mathrm{H}, \mathrm{CH}_{3}, \mathrm{~J}=6.9 \mathrm{~Hz}\right), 1.32\left(\mathrm{t}, 12 \mathrm{H}, \mathrm{CH}_{3}, \mathrm{~J}=\right.$ $6.5 \mathrm{~Hz}), 1.52\left(\mathrm{~m}, 12 \mathrm{H}, \mathrm{CH}_{2}\right), 1.82\left(\mathrm{~m}, 12 \mathrm{H}, \mathrm{CH}_{2}\right), 3.33$ (d, $\left.4 \mathrm{H}, \mathrm{CH}_{2}-\mathrm{P}, \mathrm{J}_{\mathrm{H}-\mathrm{P}}=21.9 \mathrm{~Hz}\right), 4.09\left(\mathrm{~m}, 20 \mathrm{H}, \mathrm{CH}_{2}-\mathrm{O}\right), 7.05(\mathrm{~s}$, $2 \mathrm{H}, \mathrm{H}-\mathrm{Ar}), 7.24\left(\mathrm{~d}, 2 \mathrm{H},=\mathrm{CH}, \mathrm{J}_{\text {trans }}=16.8 \mathrm{~Hz}\right), 7.24(\mathrm{~s}, 2 \mathrm{H}$, $\mathrm{H}-\mathrm{Ar}), 7.29$ (s, 2H, H-Ar), 7.33 (d, 2H, =CH, J $7.45\left(\mathrm{~d}, 2 \mathrm{H},=\mathrm{CH}, \mathrm{J}_{\text {trans }}=16.2 \mathrm{~Hz}\right), 7.49\left(\mathrm{~m}, 8 \mathrm{H}, \mathrm{H}-\mathrm{Ar}_{\mathrm{ant}}, \mathrm{J}=\right.$ $6.2 \mathrm{~Hz}), 7.96\left(\mathrm{~d}, 1 \mathrm{H},=\mathrm{CH}, \mathrm{J}_{\text {trans }}=16.5 \mathrm{~Hz}\right), 8.04(\mathrm{~d}, 1 \mathrm{H}$, $\left.=\mathrm{CH}, \mathrm{J}_{\text {trans }}=16.8 \mathrm{~Hz}\right), 8.49\left(\mathrm{~m}, 8 \mathrm{H}, \mathrm{H}-\mathrm{Ar}_{\mathrm{ant}}, \mathrm{J}=8.4 \mathrm{~Hz}\right) .13 \mathrm{C}$ NMR $\left(\mathrm{CDCl}_{3}\right), \delta(\mathrm{ppm}): 13.85\left(\mathrm{CH}_{3}\right), 16.4\left(\mathrm{CH}_{3}\right), 19.4$ $\left(\mathrm{CH}_{2}\right), 31.5\left(\mathrm{P}-\mathrm{CH}_{2}\right), 31.6\left(\mathrm{CH}_{2}\right), 68.9\left(\mathrm{O}-\mathrm{CH}_{2}\right), 69.2(\mathrm{O}-$ $\left.\mathrm{CH}_{2}\right), 110.6\left(\mathrm{HC}_{\mathrm{ar}}\right), 111.3\left(\mathrm{HC}_{\mathrm{ar}}\right), 115.7\left(\mathrm{HC}_{\mathrm{ar}}\right), 121.1\left(\mathrm{C}_{\mathrm{ar}}\right)$, $121.3\left(\mathrm{C}_{\mathrm{ar}}\right), 125.1\left(\mathrm{HC}_{\mathrm{ar}}\right), 125.5(=\mathrm{CH}), 125.9(=\mathrm{CH}), 126.6$
$\left(\mathrm{HC}_{\text {ant }}\right), 127.1\left(\mathrm{C}_{\mathrm{ipso}}\right), 129.6\left(\mathrm{C}_{\mathrm{ant}}\right), 132.8\left(\mathrm{C}_{\mathrm{ipso}}\right), 132.9\left(\mathrm{C}_{\mathrm{ipso}}\right)$, $133.1\left(\mathrm{C}_{\text {ipso }}\right), 133.3\left(\mathrm{C}_{\text {ipso }}\right), 150.7$ (O-C), 151.3 (O-C). FAB+ Ms $(\mathrm{m} / \mathrm{z}): 1418[\mathrm{M}]+$. Calc. for $\mathrm{C}_{88} \mathrm{H}_{108} \mathrm{O}_{12} \mathrm{P}_{2}: \mathrm{C}, 74.45 ; \mathrm{H}$, 7.67 (\%). Found: C, 74.43; H, $7.69(\%)$.

\section{1-Pyrene-methanol (13)}

A solution of 1-pyrenecarboxaldehyde 13 (5 g, 21.7 $\mathrm{mmol}$ ) in $50 \mathrm{~mL}$ of dry THF was added dropwise to a lithium aluminum hydride $(0.82 \mathrm{~g}, 10.8 \mathrm{mmol})$ in $50 \mathrm{~mL}$ of dry THF. The reaction was carried out at $0{ }^{\circ} \mathrm{C}$ for $4 \mathrm{~h}$. After this time, $10 \mathrm{~mL}$ of water were added and the reaction mixture was filtered in Celite ${ }^{\circledR}$. The solvent was evaporated and the residue was dissolved in dichloromethane. The resulting solution was dried over sodium sulfate, filtered and the product was purified by silica gel chromatography using hexane as an eluent to afford 1-pyrene-methanol (15) as a yellow powder, yield 4.9 g, 21.11 mmol (97 \%), m.p. 123-125 ${ }^{\circ}$ C. FTIR. (pellet, $\mathrm{KBr}, \mathrm{cm}^{-1}$ ): 3279, 1590, 1181, 1064, 1008, 841, 706. UV-vis $\left(\mathrm{CHCl}_{3}, \mathrm{~nm}\right) \lambda_{\text {max }}: 344,328,315,278,267,257,244$. ${ }^{1} \mathrm{H}$ NMR $\left(200 \mathrm{MHz}, \mathrm{CDCl}_{3}\right), \delta(\mathrm{ppm}): 1.95(\mathrm{~s}, 1 \mathrm{H}, \mathrm{OH})$, $5.31\left(\mathrm{~s}, 2 \mathrm{H}, \mathrm{CH}_{2}\right), 7.93-8.29\left(\mathrm{~m}, 9 \mathrm{H}, \mathrm{H}_{p y r}\right) .{ }^{13} \mathrm{C}$ NMR (CD$\left.\mathrm{Cl}_{3}\right), \delta(\mathrm{ppm}): 63.7\left(\mathrm{CH}_{2}\right), 122.9$ (Ar), 124.6 (Ar), 124.8 $\left(\mathrm{C}_{\mathrm{ar}}\right), 125.2(\mathrm{Ar}), 125.9(\mathrm{Ar}), 127.3$ (Ar), 127.8 (Ar), 128.6 $\left(\mathrm{C}_{\mathrm{ar}}\right), 130.7\left(\mathrm{C}_{\mathrm{ar}}\right), 131.1\left(\mathrm{C}_{\mathrm{ar}}\right), 133.6\left(\mathrm{C}_{\mathrm{ipso}}\right) . \mathrm{EM}: \mathrm{m} / \mathrm{z}: 232$ $[\mathrm{M}]^{+}$. Calc. for $\mathrm{C}_{17} \mathrm{H}_{12} \mathrm{O}: \mathrm{C}, 87.90 ; \mathrm{H}, 5.21$ (\%); Found: C, 87.91; H, $5.20(\%)$.

\section{1-Chlromethyl-pyrene (14)}

A solution of pyridine $(1 \mathrm{~mL}, 14.0 \mathrm{mmol})$ and $\mathrm{SOCl}_{2}$ (1.45 mL, $14.0 \mathrm{mmol}$ ) in $100 \mathrm{~mL}$ of dry $\mathrm{CH}_{2} \mathrm{Cl}_{2}$ was added to $13(4.1 \mathrm{~g}, 17.7 \mathrm{mmol})$, and then this mixture was cooled to $-10^{\circ} \mathrm{C}$. The reaction was carried out in nitrogen atmosphere for $7 \mathrm{~h}$. After this, the solvent was evaporated and the resulting oil was dried and purified by silica gel chromatography using a mixture of hexane-dichloromethane 2:1 as an eluent to afford 1-chloromethil pyrene (16) as a brownish powder, yield $4.3 \mathrm{~g}, 17.19 \mathrm{mmol}(99 \%)$, m.p. $148-149{ }^{\circ} \mathrm{C}$. FTIR. (pellet, $\left.\mathrm{KBr}, \mathrm{cm}^{-1}\right): 3042,1591,1254,842,688$. UV-vis $\left(\mathrm{CHCl}_{3}\right.$, nm) $\lambda_{\max }: 378,349,333,280,269,245 .{ }^{1} \mathrm{H}$ NMR $(200 \mathrm{MHz}$, $\left.\mathrm{CDCl}_{3}\right) \delta(\mathrm{ppm}): 5.31\left(\mathrm{~s}, 2 \mathrm{H}, \mathrm{CH}_{2}\right), 7.97-8.38\left(\mathrm{~m}, 9 \mathrm{H}, \mathrm{H}_{p y r}\right)$. ${ }^{13} \mathrm{C} \mathrm{NMR}\left(\mathrm{CDCl}_{3}\right), \delta(\mathrm{ppm}): 44.7\left(\mathrm{CH}_{2}\right), 122.7(\mathrm{Ar}), 124.5$ $(\mathrm{Ar}), 124.7\left(\mathrm{C}_{\mathrm{ar}}\right), 125.0\left(\mathrm{C}_{\mathrm{ar}}\right), 125.6(\mathrm{Ar}), 126.1(\mathrm{Ar}), 127.2$ (Ar), 127.6 (Ar), 127.9 (Ar), 128.3 (Ar), $129.11\left(\mathrm{C}_{\mathrm{ar}}\right), 130.2$ $\left(\mathrm{C}_{\mathrm{ar}}\right), 130.6\left(\mathrm{C}_{\mathrm{ar}}\right), 131.9\left(\mathrm{C}_{\mathrm{ipso}}\right)$. EM: m/z: $250[\mathrm{M}]^{+}$. Calc. for $\mathrm{C}_{17} \mathrm{H}_{11} \mathrm{Cl}$ : C, 81.44; H, 4.42; Cl, 14.14 (\%); found: C, 81.44; $\mathrm{H}, 4.42(\%)$.

\section{1-Pyrenephosphonate (15)}

Triethyl phosphite $(4 \mathrm{ml}, 24.07 \mathrm{mmol})$ and $16(5.0 \mathrm{~g}$, $19.94 \mathrm{mmol}$ ) were stirred at $140{ }^{\circ} \mathrm{C}$ for $5 \mathrm{~h}$. The reaction mixture was cooled at room temperature and purified by silica gel chromatography using ethyl acetate as an eluent to afford 1-pyrenephosphonate (17) as yellow pale oil, yield $10.3 \mathrm{~g}, 19.9 \mathrm{mmol}$ (98 \%). FTIR. (pellet, $\mathrm{KBr}, \mathrm{cm}^{-1}$ ): 3042 , 2982, 2906, 1243, 1058, 1024, 960, 790, 616. UV-vis $\left(\mathrm{CHCl}_{3}, \mathrm{~nm}\right) \lambda_{\max }: 376,345,329,314,278,267,257,244$. ${ }^{1} \mathrm{H}$ NMR $\left(200 \mathrm{MHz}, \mathrm{CDCl}_{3}\right), \delta(\mathrm{ppm}): 1.15\left(\mathrm{t}, 6 \mathrm{H}, \mathrm{CH}_{3}, J=\right.$ $6.7 \mathrm{~Hz}), 3.91\left(\mathrm{~m}, 6 \mathrm{H}, \mathrm{H}_{2} \mathrm{C}-\mathrm{P}, \mathrm{H}_{2} \mathrm{C}-\mathrm{O}\right), 7.96-8.35(\mathrm{~m}, 9 \mathrm{H}$, $\left.\mathrm{H}_{\text {pyr }}\right) .{ }^{13} \mathrm{C} \mathrm{NMR}\left(\mathrm{CDCl}_{3}\right), \delta(\mathrm{ppm}): 16.4\left(\mathrm{CH}_{3}\right), 30.1$ and 32.8 $\left(\mathrm{H}_{2} \mathrm{C}-\mathrm{P}\right), 62.2\left(\mathrm{H}_{2} \mathrm{C}-\mathrm{O}\right), 123.7$ ( $\left.\mathrm{Ar}\right), 124.7\left(\mathrm{C}_{\mathrm{ar}}\right), 124.8$ (Ar), 124.9 (Ar), 125.1 (Ar), $125.6\left(\mathrm{C}_{\mathrm{ar}}\right), 125.9$ (Ar), 127.1 (Ar), 127.3 (Ar), 127.5 (Ar), $128.7\left(\mathrm{C}_{\mathrm{ar}}\right), 129.2\left(\mathrm{C}_{\mathrm{ar}}\right), 129.4\left(\mathrm{C}_{\mathrm{ar}}\right)$, 
$130.5\left(\mathrm{C}_{\mathrm{ar}}\right), 131.2\left(\mathrm{C}_{\mathrm{ipso}}\right)$. EM: m/z: $352[\mathrm{M}]^{+}$. Calc. for $\mathrm{C}_{21} \mathrm{H}_{21} \mathrm{O}_{3} \mathrm{P}: \mathrm{C}, 71.58 ; \mathrm{H}, 6.01$; P, 8.79 (\%); found: C, 71.56; $\mathrm{H}, 6.01 ; \mathrm{P}, 8.77$ (\%).

\section{(E)-10-(2-(Pyren-1-yl)vinyl)anthracene-9-carbaldehyde (16)}

A solution of 9,10-anthracenedicarboxaldehyde 2 (3.0 g, $8.54 \mathrm{mmol}), 1$-pryrenephosphonate $\mathbf{1 5}(2 \mathrm{~g}, 8.54 \mathrm{mmol})$ in $100 \mathrm{ml}$ of dry THF was stirred under nitrogen at room temperature, and tert-BuOK $(1.04 \mathrm{~g}, 8.54 \mathrm{mmol})$ was added. The solution was stirred for $10 \mathrm{~h}$, then water $(2 \mathrm{ml})$ was added. The solvent was evaporated under vacuum and the residue was purified by silica gel chromatography using a mixture of 60:40 hexane-dichloromethane as an eluent to afford (E)-10(2-(pyren-1-yl)vinyl)anthracene-9-carbaldehyde (16) as an orange powder, yield $2.31 \mathrm{~g}, 5.34 \mathrm{mmol}$ (63\%), m.p. $>300$ ${ }^{\circ} \mathrm{C}$. FTIR. (pellet, $\mathrm{KBr}, \mathrm{cm}^{-1}$ ): 3040, 1671, 1519, 1409, 1257 , $1109,843,754,618$. UV-vis $\left(\mathrm{CHCl}_{3}, \mathrm{~nm}\right) \lambda_{\max }: 435,361$, 270, 241. ${ }^{1} \mathrm{H}$ NMR $\left(200 \mathrm{MHz}, \mathrm{CDCl}_{3}\right), \delta(\mathrm{ppm}): 7.57(\mathrm{~d}, 1 \mathrm{H}$, $\left.=\mathrm{CH}, J_{\text {trans }}=15 \mathrm{~Hz}\right), 7.58\left(\mathrm{~s}, 1 \mathrm{H}, \mathrm{H}_{p y r}\right), 7.72(\mathrm{~d}, 1 \mathrm{H},=\mathrm{CH}$, $\left.J_{\text {trans }}=14.7 \mathrm{~Hz}\right), 7.72\left(\mathrm{~s}, 1 \mathrm{H}, \mathrm{H}_{p y r}\right), 8.05\left(\mathrm{dd}, 2 \mathrm{H}, \mathrm{H}_{a n t}, J=7.5\right.$, $10.2 \mathrm{~Hz}), 8.13\left(\mathrm{~s}, 2 \mathrm{H}, \mathrm{H}_{p y r}\right), 8.20\left(\mathrm{dd}, 2 \mathrm{H}, \mathrm{H}_{a n t}, J=7.8,11.1\right.$ $\mathrm{Hz}), 8.31\left(\mathrm{~d}, 2 \mathrm{H}, \mathrm{H}_{p y r}, J=8.1 \mathrm{~Hz}\right), 8.37\left(\mathrm{~d}, 2 \mathrm{H}, \mathrm{H}_{p y r}, J=9.3\right.$ $\mathrm{Hz}), 8.61\left(\mathrm{~d}, 2 \mathrm{H}, \mathrm{H}_{a n t}, J=8.7 \mathrm{~Hz}\right), 8.63\left(\mathrm{~d}, 1 \mathrm{H}, \mathrm{H}_{p y r}, J=7.8\right.$ $\mathrm{Hz}), 8.05\left(\mathrm{~d}, 2 \mathrm{H}, \mathrm{H}_{\text {ant }}, J=9.0 \mathrm{~Hz}\right), 11.58(\mathrm{~s}, 1 \mathrm{H}, \mathrm{HC}=\mathrm{O}) .{ }^{13} \mathrm{C}$ NMR $\left(\mathrm{CDCl}_{3}\right), \delta(\mathrm{ppm}): 122.8(\mathrm{Ar}), 1123.8(\mathrm{Ar}), 123.9(\mathrm{Ar})$, 125.2 (Ar), $125.3(=\mathrm{CH}), 125.6(\mathrm{Ar}), 125.8(\mathrm{Ar}), 126.2(\mathrm{Ar})$, 127.1 (Ar), $127.4(\mathrm{Ar}), 127.8(\mathrm{Ar}), 128.2(\mathrm{Ar}), 128.7(\mathrm{Ar})$, $128.2(\mathrm{Ar}), 129.6\left(\mathrm{C}_{\mathrm{ar}}\right), 131.2\left(\mathrm{C}_{\mathrm{ar}}\right), 131.6\left(\mathrm{C}_{\mathrm{ipso}}\right), 131.9\left(\mathrm{C}_{\mathrm{ip}-}\right.$ so), $193.1(\mathrm{HC}=\mathrm{O})$. EM: m/z: $432[\mathrm{M}]^{+}$. Calc. for $\mathrm{C}_{33} \mathrm{H}_{20}$ : C, 91.64; H, 4.66 (\%); Found; C, 91.64; H, 4.66 (\%).

10-((E)-2,5-Dibutoxy-4-((E)-2-(10-((E)-2-(pyren-1-yl)vinyl) anthracen-9-yl)vinyl)styryl)anthracene-9-carbaldehyde (17)

A solution of $11(0.63 \mathrm{~g}, 0.92 \mathrm{mmol})$ and 1pryrenephosphonate $15(2 \mathrm{~g}, 8.54 \mathrm{mmol})$ of in $20 \mathrm{ml}$ of dry THF was stirred under nitrogen at room temperature, and tert-BuOK $0.113 \mathrm{~g}(0.92 \mathrm{mmol})$ was added. The reaction was stirred and heated at $60{ }^{\circ} \mathrm{C}$ overnight, and then water $(2 \mathrm{ml})$ was added, the solvent was evaporated under vacuum and the residue was purified by silica gel chromatography using a mixture of 60:40 hexane-dichloromethane as an eluent to afford 10-((E)-2,5-dibutoxy-4-( $(E)-2-(10-((E)-2$-(pyren-1yl)vinyl)anthracen-9-yl)vinyl)styryl)anthracene-9-carbaldehyde (17) as a orange powder, yield $0.45 \mathrm{~g}, 0.51 \mathrm{mmol}(56$ $\%$ ), m.p. $>300{ }^{\circ} \mathrm{C}$. FTIR. (pellet, $\mathrm{KBr}, \mathrm{cm}^{-1}$ ): 3052,2960 , 2868, 1672, 1441, 1265, 1180, 1034, 752. UV-vis $\left(\mathrm{CHCl}_{3}\right.$, nm) $\lambda_{\max }: 437,254 .{ }^{1} \mathrm{H}$ NMR $\left(300 \mathrm{MHz}, \mathrm{CDCl}_{3}\right), \delta(\mathrm{ppm})$ : $0.95\left(\mathrm{t}, 6 \mathrm{H}, \mathrm{CH}_{3}, J=6.7 \mathrm{~Hz}\right), 1.53\left(\mathrm{~m}, 4 \mathrm{H}, \mathrm{CH}_{2}\right), 1.83(\mathrm{~m}$, $\left.4 \mathrm{H}, \mathrm{CH}_{2}\right), 4.17\left(\mathrm{t}, 4 \mathrm{H}, \mathrm{CH}_{2}-\mathrm{O}, J=6.6 \mathrm{~Hz}\right), 7.35(\mathrm{~d}, 1 \mathrm{H},=\mathrm{CH}$, $\left.J_{\text {trans }}=16.2 \mathrm{~Hz}\right), 7.42(\mathrm{~s}, 2 \mathrm{H}, \operatorname{Ar}-\mathrm{H}), 7.52\left(\mathrm{~d}, 1 \mathrm{H},=\mathrm{CH}, J_{\text {trans }}=\right.$ $16.2 \mathrm{~Hz}), 7.53\left(\mathrm{~d}, 1 \mathrm{H},=\mathrm{CH}, J_{\text {trans }}=16.2 \mathrm{~Hz}\right), 7.53\left(\mathrm{~s}, 1 \mathrm{H}, \mathrm{H}_{p-}\right.$ $\left.{ }_{y r}\right), 7.55\left(\mathrm{~d}, 1 \mathrm{H},=\mathrm{CH}, J_{\text {trans }}=16.2 \mathrm{~Hz}\right), 7.61-7.74(\mathrm{~m}, 2 \mathrm{H}$, $\left.\mathrm{H}_{a n t}\right), 7.75\left(\mathrm{~s}, 2 \mathrm{H}, \mathrm{H}_{p y r}\right), 7.96\left(\mathrm{~d}, 1 \mathrm{H},=\mathrm{CH}, J_{\text {trans }}=16.2 \mathrm{~Hz}\right)$, $7.96\left(\mathrm{~m}, 4 \mathrm{H}, \mathrm{H}_{a n t}\right), 8.05\left(\mathrm{~m}, 4 \mathrm{H}, \mathrm{H}_{a n t}\right), 8.08(\mathrm{~d}, 1 \mathrm{H},=\mathrm{CH}, J-$ trans $=16.2 \mathrm{~Hz}), 8.06-811\left(\mathrm{~m}, 2 \mathrm{H}, \mathrm{H}_{a n t}\right), 8.10\left(\mathrm{~s}, 2 \mathrm{H}, \mathrm{H}_{p y r}\right)$, $8.44\left(\mathrm{~d}, 2 \mathrm{H}, \mathrm{H}_{p y r}, J=7.7 \mathrm{~Hz}\right), 8.43-8.58\left(\mathrm{~m}, 2 \mathrm{H}, \mathrm{H}_{a n t}\right), 9.03$ $\left(\mathrm{d}, 2 \mathrm{H}, \mathrm{H}_{\text {ant }}, J=9.0 \mathrm{~Hz}\right), 11.55(\mathrm{~s}, 1 \mathrm{H}, \mathrm{HC}=\mathrm{O}) .{ }^{13} \mathrm{C} \mathrm{NMR}$ $\left(\mathrm{CDCl}_{3}\right), \delta(\mathrm{ppm}): 13.8\left(\mathrm{CH}_{3}\right), 19.4\left(\mathrm{CH}_{2}\right), 31.5\left(\mathrm{CH}_{2}\right), 69.2$ $\left(\mathrm{CH}_{2}-\mathrm{O}\right), 111.2(\mathrm{Ar}), 123.8(\mathrm{Ar}), 125.1(\mathrm{Ar}), 125.3(=\mathrm{CH})$, 125.5 (Ar), 126.2 (Ar), $126.3\left(\mathrm{C}_{\mathrm{ipso}}\right), 126.6(=\mathrm{CH}), 127.1$ $(=\mathrm{CH}), 128.7$ (Ar), $129.6(=\mathrm{CH}), 131.5\left(\mathrm{C}_{\mathrm{ar}}\right), 131.9\left(\mathrm{C}_{\mathrm{ar}}\right)$,
$132.8\left(\mathrm{C}_{\mathrm{ipso}}\right), 133.3\left(\mathrm{C}_{\mathrm{ipso}}\right), 151.3$ (Ar-O). EM: m/z: $880[\mathrm{M}]^{+}$ . Calc. for $\mathrm{C}_{65} \mathrm{H}_{52} \mathrm{O}_{3}$ : C, 88.60; H, 5.95 (\%); Found; C, 88.60; H, 5.95 .

\section{Diethyl 2,5-dibutoxy-4-((E)-2-(10-((E)-2,5-dibutoxy-4-((E)- 2-(10-((E)-2,5-dibutoxy-4-((E)-2-(pyren-1-yl)vinyl)styryl) anthracen-9-yl)vinyl)styryl)anthracen-9-yl)vinyl)benzylph- osphonate (18)}

A solution of $17(0.57 \mathrm{~g}, 0.40 \mathrm{mmol})$, and 1-pyrene carboxaldehyde $13(0.11 \mathrm{~g}, 0.32 \mathrm{mmol})$ in $100 \mathrm{ml}$ of dry THF was stirred under nitrogen and cooled at room temperature, and tert- $\mathrm{BuOK}(0.11 \mathrm{~g}, 0.98 \mathrm{mmol})$ was added. The solution was stirred for $10 \mathrm{~h}$, and then water $(2 \mathrm{ml})$ was added, the solvent was evaporated under vacuum and the residue was purified by silica gel chromatography using a mixture of 60:40 hexane-dichloromethane as an eluent to afford diethyl 2,5-dibutoxy-4-((E)-2-(10-( $(E)-2,5$-dibutoxy4-((E)-2-(10-((E)-2,5-dibutoxy-4-((E)-2-(pyren-1-yl)vinyl) styryl)anthracen-9-yl)vinyl)styryl)anthracen-9-yl)vinyl)benzylphosphonate (18) as an orange powder, yield $0.52 \mathrm{~g}, 0.34$ mmol (86\%), m.p. $>300{ }^{\circ} \mathrm{C}$. FTIR. (pellet, $\mathrm{KBr}, \mathrm{cm}^{-1}$ ): 3043 , 2958, 2925, 2866, 1726, 1497, 1382, 1202, 1029, 756. UVvis $\left(\mathrm{CHCl}_{3}, \mathrm{~nm}\right) \lambda_{\max }: 677,440,264 .{ }^{1} \mathrm{H}$ NMR $(300 \mathrm{MHz}$, $\left.\mathrm{CDCl}_{3}\right), \delta(\mathrm{ppm}): 0.96\left(\mathrm{~m}, 18 \mathrm{H}, \mathrm{CH}_{3}\right), 1.32\left(\mathrm{t}, 6 \mathrm{H}, \mathrm{CH}_{3}\right.$, $J=6.5 \mathrm{~Hz}), 1.56\left(\mathrm{~m}, 12 \mathrm{H}, \mathrm{CH}_{2}\right), 1.85\left(\mathrm{~m}, 12 \mathrm{H}, \mathrm{CH}_{2}\right), 3.33$ $\left(\mathrm{d}, 2 \mathrm{H}, \mathrm{CH}_{2}-\mathrm{P}, J_{\mathrm{H}-\mathrm{P}}=21.9 \mathrm{~Hz}\right), 4.13\left(\mathrm{~m}, 16 \mathrm{H}, \mathrm{CH}_{2}-\mathrm{O}\right), 7.05$ $(\mathrm{s}, 1 \mathrm{H}, \mathrm{H}-\mathrm{Ar}), 7.05(\mathrm{~s}, 1 \mathrm{H}, \mathrm{H}-\mathrm{Ar}), 7.25\left(\mathrm{~d}, 1 \mathrm{H},=\mathrm{CH}, J_{\text {trans }}=\right.$ $17.1 \mathrm{~Hz}), 7.29$ (s, 1H, H-Ar), 7.31 (s, 1H, H-Ar), 7.36 $\left(\mathrm{d}, 1 \mathrm{H},=\mathrm{CH}, J_{\text {trans }}=16.5 \mathrm{~Hz}\right), 7.37\left(\mathrm{br}, 2 \mathrm{H}, \mathrm{H}-\mathrm{Ar}, \mathrm{H}_{\mathrm{Py}}\right), 7.43$ $(\mathrm{s}, 1 \mathrm{H}, \mathrm{H}-\mathrm{Ar}), 7.47\left(\mathrm{~d}, 1 \mathrm{H},=\mathrm{CH}, J_{\text {trans }}=16.8 \mathrm{~Hz}\right), 7.52(\mathrm{~m}$, $\left.10 \mathrm{H}, \mathrm{H}_{\text {Py }}, \mathrm{H}_{\text {Ant }}\right), 7.75\left(\mathrm{~d}, 1 \mathrm{H},=\mathrm{CH}, J_{\text {trans }}=15.9 \mathrm{~Hz}\right), 7.96(\mathrm{~d}$, $\left.1 \mathrm{H},=\mathrm{CH}, J_{\text {trans }}=17.1 \mathrm{~Hz}\right), 8.06\left(\mathrm{~m}, 3 \mathrm{H}, \mathrm{H}_{\mathrm{Py}}\right), 8.06(\mathrm{~d}, 1 \mathrm{H}$, $\left.=\mathrm{CH}, J_{\text {trans }}=16.8 \mathrm{~Hz}\right), 8.17\left(\mathrm{~d}, 1 \mathrm{H},=\mathrm{CH}, J_{\text {trans }}=16.2 \mathrm{~Hz}\right)$, $8.17\left(\mathrm{~m}, 3 \mathrm{H}, \mathrm{H}_{\mathrm{Py}}\right), 8.34\left(\mathrm{~d}, 1 \mathrm{H},=\mathrm{CH}, J_{\text {trans }}=16.2 \mathrm{~Hz}\right), 8.44$ $\left(\mathrm{d}, 1 \mathrm{H},=\mathrm{CH}, J_{\text {trans }}=15.9 \mathrm{~Hz}\right), 8.54\left(\mathrm{~m}, 8 \mathrm{H}, \mathrm{H}_{\text {Ant }}\right), 8.55(\mathrm{~d}$, $\left.1 \mathrm{H},=\mathrm{CH}, J_{\text {trans }}=16.8 \mathrm{~Hz}\right) .{ }^{13} \mathrm{C}$ NMR $\left(\mathrm{CDCl}_{3}\right), \delta(\mathrm{ppm}): 13.8$ $\left(\mathrm{CH}_{3}\right), 16.4\left(\mathrm{CH}_{3}\right), 19.4\left(\mathrm{CH}_{2}\right), 31.6\left(\mathrm{P}-\mathrm{CH}_{2}\right), 61.9\left(\mathrm{O}-\mathrm{CH}_{2}\right)$, $69.2\left(\mathrm{O}-\mathrm{CH}_{2}\right), 123.7\left(\mathrm{HC}_{\mathrm{ar}}\right), 125.1\left(\mathrm{HC}_{\mathrm{ar}}\right), 125.9(=\mathrm{CH})$, $126.3(=\mathrm{CH}), 126.6\left(\mathrm{HC}_{\mathrm{Py}}\right), 127.1\left(\mathrm{HC}_{\mathrm{ant}}\right), 127.5\left(\mathrm{HC}_{\mathrm{ant}}\right)$, $128.4\left(\mathrm{C}_{\mathrm{ipso}}\right), 129.6\left(\mathrm{C}_{\mathrm{ant}}\right), 130.7\left(\mathrm{C}_{\mathrm{ipso}}\right), 133.0\left(\mathrm{C}_{\mathrm{ipso}}\right), 131.6$ $\left(\mathrm{C}_{\mathrm{ipso}}\right), 132.8\left(\mathrm{HC}_{\mathrm{py}}\right), 133.3\left(\mathrm{C}_{\mathrm{py}}\right), 135.1\left(\mathrm{C}_{\mathrm{py}}\right), 150.3(\mathrm{O}-\mathrm{C})$, 151.4 (O-C). EM: m/z: $1495[\mathrm{M}]^{+}$. Calc. for $\mathrm{C}_{101} \mathrm{H}_{107} \mathrm{O}_{9} \mathrm{P}: \mathrm{C}$, 81.09; H, 7.21 (\%); Found; C, 81.10; H, 4.66 (\%).

10-((E)-2,5-Dibutoxy-4-((E)-2-(10-((E)-2,5-dibutoxy-4-((E)2-(10-((E)-2,5-dibutoxy-4-((E)-2-(pyren-1-yl)vinyl)styryl) anthracen-9-yl)vinyl)styryl)anthracen-9-yl)vinyl)styryl)anthracene-9-carbaldehyde (19)

A solution of 9,10-anthracenedicarboxaldehyde $2(0.10 \mathrm{~g}$, $0.42 \mathrm{mmol})$ and $18(0.52 \mathrm{~g}, 0.34 \mathrm{mmol})$ in $100 \mathrm{ml}$ of dry THF was stirred under nitrogen at room temperature, tertBuOK $(0.06 \mathrm{~g}, 0.53 \mathrm{mmol})$ was added. The solution was stirred for $10 \mathrm{~h}$, and then water $(2 \mathrm{ml})$ was added, the solvent was evaporated under vacuum and the residue was purified by silica gel chromatography using a mixture of 60:40 hexane-dichloromethane as an eluent to afford 10-((E)-2,5dibutoxy-4-((E)-2-(10-((E)-2,5-dibutoxy-4-((E)-2-(10-((E)2,5-dibutoxy-4-((E)-2-(pyren-1-yl)vinyl)styryl)anthracen-9yl)vinyl)styryl)anthracen-9-yl)vinyl)styryl) anthracene-9carbaldehyde (19) as an orange powder, yield $0.53 \mathrm{~g}, 0.33$ mmol (98 \%), m.p. $>300$ oC. FTIR. (pellet, $\mathrm{KBr}, \mathrm{cm}-1$ ): 3046, 2955, 2930, 2868, 1627, 1413, 1383, 1198, 1029, 977, 
755. UV-vis $\left(\mathrm{CH}_{2} \mathrm{Cl}_{2}, \mathrm{~nm}\right) \lambda_{\max }: 224,260,348,449.1 \mathrm{H}$ NMR (300 MHz, acetone- $\left.\mathrm{d}_{6}\right), \delta(\mathrm{ppm}): 0.974\left(\mathrm{~m}, 18 \mathrm{H}, \mathrm{CH}_{3}\right)$, $1.53\left(\mathrm{~m}, 12 \mathrm{H}, \mathrm{CH}_{3}\right), 1.84\left(\mathrm{~m}, 12 \mathrm{H}, \mathrm{CH}_{2}\right), 4.28(\mathrm{~m}, 12 \mathrm{H}$, $\left.\mathrm{CH}_{2}-\mathrm{O}\right), 6.99(\mathrm{~d}, 2 \mathrm{H}, \mathrm{H}-\mathrm{Ar}, J=8.2,8.0 \mathrm{~Hz}), 7.41(\mathrm{~d}, 1 \mathrm{H}$, $\left.=\mathrm{CH}, J_{\text {trans }}=16.5 \mathrm{~Hz}\right), 7.46-7.64\left(\mathrm{br}, 10 \mathrm{H}, \mathrm{H}_{\mathrm{Ar}},=\mathrm{CH}, \mathrm{H}_{\mathrm{Ant}}\right)$, 7.85-7.81 (br, 7H, =CH, H-Ar, $\left.\mathrm{H}_{\text {Ant }}\right), 7.91\left(\mathrm{~d}, 2 \mathrm{H}, \mathrm{H}_{\mathrm{Py}}, J=7.7\right.$ $\mathrm{Hz}), 7.96\left(\mathrm{~d}, 1 \mathrm{H},=\mathrm{CH}, J_{\text {trans }}=15.9 \mathrm{~Hz}\right), 7.99(\mathrm{~d}, 1 \mathrm{H},=\mathrm{CH}$, $\left.J_{\text {trans }}=16.2 \mathrm{~Hz}\right), 8.06\left(\mathrm{br}, 2 \mathrm{H}, \mathrm{H}_{\mathrm{Py}}\right), 8.12\left(\mathrm{~d}, 1 \mathrm{H},=\mathrm{CH}, J_{\text {trans }}=\right.$ $15.9 \mathrm{~Hz},), 8.12\left(\mathrm{br}, 2 \mathrm{H}, \mathrm{H}_{\mathrm{Py}}\right), 8.17-8.18\left(\mathrm{br}, 5 \mathrm{H}, \mathrm{H}_{\mathrm{Ant}}, \mathrm{H}_{\mathrm{Py}}\right.$ ), $8.24\left(\mathrm{~d}, 1 \mathrm{H},=\mathrm{CH}, J_{\text {trans }}=16.8 \mathrm{~Hz},\right), 8.25\left(\mathrm{~d}, 1 \mathrm{H},=\mathrm{CH}, J_{\text {trans }}=\right.$ $16.2 \mathrm{~Hz}), 8.43\left(\mathrm{~d}, 4 \mathrm{H}, \mathrm{H}_{\text {Ant }}, J=9.3 \mathrm{~Hz}\right.$,), 8.51-8.66 (br, 7H, $\left.\mathrm{H}_{\mathrm{Ant}},=\mathrm{CH}, \mathrm{H}_{\mathrm{Py}}\right), 8.87\left(\mathrm{br}, 2 \mathrm{H}, \mathrm{H}_{\mathrm{Ant}}\right), 9.56\left(\mathrm{br}, 2 \mathrm{H}, \mathrm{H}_{\mathrm{Ant}}\right)$, $10.87(\mathrm{~s}, 1 \mathrm{H}, \mathrm{HC}=\mathrm{O}) .13 \mathrm{C}$ NMR (acetone- $\left.\mathrm{d}_{6}\right), \delta(\mathrm{ppm}): 14.1$ $\left(\mathrm{CH}_{3}\right), 20.1\left(\mathrm{CH}_{2}\right), 32.3\left(\mathrm{CH}_{2}\right), 61.9\left(\mathrm{O}_{-} \mathrm{CH}_{2}\right), 111.5\left(\mathrm{HC}_{\mathrm{ar}}\right)$, $111.9\left(\mathrm{HC}_{\mathrm{ar}}\right), 124.9\left(\mathrm{HC}_{\mathrm{ar}}\right), 125.4(=\mathrm{CH}), 125.5\left(\mathrm{C}_{\mathrm{ar}}\right), 126.1$ $(=\mathrm{CH}), 126.4\left(\mathrm{HC}_{\mathrm{ar}}\right), 126.8\left(\mathrm{HC}_{\mathrm{ar}}\right), 127.3\left(\mathrm{HC}_{\mathrm{ar}}\right), 127.8$ $\left(\mathrm{HC}_{\mathrm{ar}}\right), 128.3\left(\mathrm{HC}_{\mathrm{ar}}\right), 128.8\left(\mathrm{HC}_{\mathrm{ar}}\right), 129.6\left(\mathrm{C}_{\mathrm{ar}}\right), 130.5\left(\mathrm{C}_{\mathrm{ar}}\right)$, $132.4\left(\mathrm{C}_{\mathrm{ar}}\right), 133.5\left(\mathrm{C}_{\mathrm{ar}}\right), 134.2\left(\mathrm{C}_{\mathrm{ar}}\right), 135.1\left(\mathrm{C}_{\mathrm{ar}}\right), 152.2(\mathrm{O}-\mathrm{C})$. EM: m/z: 1574 [M]+. Calc. for $\mathrm{C}_{113} \mathrm{H}_{106} \mathrm{O}_{7}: \mathrm{C}, 86.11 ; \mathrm{H}, 6.78$ (\%); Found; C, 86.12; H, 4.66 (\%).

\section{X-Ray Crystallography}

Suitable crystals of compounds $3, \mathbf{4 , 1 4}$ and 15 (obtained by crystallization from $\mathrm{CH}_{2} \mathrm{Cl}_{2}$ at room temperature) were rolled in epoxy resin and mounted on a glass fiber. Bruker Apex AXS CCD area detector X-Ray diffractometer was the instrument used for the determination. The data were first reduced and corrected for absorption using psi-scans, and then solved using the program SHELL-XS. All nonhydrogen atoms were refined with anisotropic thermal parameters and the hydrogen atoms were refined at calculated positions with thermal parameters constrained to the carbon atom on which they were attached.

\section{SUPPLEMENTARY MATERIALS}

Supplementary materials can be viewed at www.bentham.org/open/toocj

\section{ACKNOWLEDGEMENTS}

This work was supported by the DGAPA (IN211408). We would also like to thank Nieves Z. S. M., Rios O. H.,
Velasco L., Huerta S. E., Patiño M. M. R., and Peña G. M. A. for technical assistance.

\section{REFERENCES}

[1] (a) Fox, M. A.; Channon, M. Photoinduced Electron Transfer, Elsevier: Amsterdam, 1988; (b) Balzani, V. Electron Transfer in Chemistry; Wiley-VCH: Weinheim, 2001.

[2] Barbara, P. F.; Meyer, T. J.; Ratner, M. A. J. Phys. Chem., 1996, $100,13148$.

[3] Newton, M. D. Chem. Rev., 1991, 91, 767.

[4] (a) Tour, J. M. Chem. Rev., 1996, 96, 537; b) Tour, J. M.; Acc. Chem. Res., 2000, 33, 791.

[5] Schwab, P. F. H.; Levin, M. D.; Michl, J. Chem. Rev., 1999, 99, 1863.

[6] Martín, R. E.; Mäder, T.; Diederich, F. Angew. Chem. Int. Ed., 1999, 38,817 .

[7] (a) Segura, J. L.; Martin, N. J. Mater. Chem., 2000, 10, 2403; (b) Lehn, J.-M. Supramolecular Chemistry, Concepts and Perspectives, VCH: Weinheim, 1995, Chapter 8.

[8] (a) Pope, M.; Kallmann, H. P.; Magnante, P. J. Chem. Phys., 1963, 38, 2042; (b) Tang, C. W.; Vanslyke, S. A. Appl. Phys. Lett., 1987, 51,913 .

[9] Nakatsuji, S.; Matsuda, K.; Uesugi, Y.; Nakashima, K.; Akiyama, S.; Katzer, G.; Fabian, W. J. Chem. Soc. Perkin Trans. 2, 1991, 861.

[10] Pfeifer, S.; Hörhold, H. H. Macromol. Chem. Phys., 1999, 200, 1870.

[11] Arbuzov, A. E. J. Russ. Phys. Chem. Soc., 1906, 38, 687.

[12] Michaelis, A.; Kaehne, R. Ber. 1898, 31, 1048.

[13] (a) Weitzel, H. P.; Bohnen, A.; Muellen, K. Makromol. Chem., 1990, 191(11), 2815; b) Weitzel, H. P.; Muellen, K. Makromol. Chem., 1990, 191(11), 2837; c) Skowronski, R.; Chodkiewicz, W.1.; Cadiot, P. Bull. Soc. Chim. Fr., 1967, 11, 4235; d) HongChen, L.; Chien-Min, T.; Guan-Hao, H.; Jyh-Ming, L. J. Polym. Sci. Part A Polym. Chem., 2006, 44(2), 783; e) Bueschel, M.; Ajayaghosh, A.; Eldo, J. Macromolecules, 2002, 35(22), 8405; f) Eldo, J.; Ajayaghosh, A. Chem. Mater., 2002, 14(1), 410; g) Liang, L.; Yi, P.; Liming, D.; Karasz, F. E. Macromolecules, 2001, 34(19), 6756; h) Eldo, J.; Arunkumar, E.; Ajayaghosh, A. Tetrahedron Lett., 2000, 41(32), 6241.

[14] Zongqiang, Y.; Songting, T.; Yingping, Z.; Benhu, F.; Zhuliang, Y.; Yongfang, L. J. Appl. Polym. Sci., 2006, 102, 3955.

[15] Kuhnert, N.; Lopez-Periago, A.; Rossignolo, M. G. Org. Biomol. Chem., 2005, 3, 524.

[16] Miller, W, M.; Amidon, W. R.; Tawney, O. P. J. Am. Chem. Soc., 1955, $77,2848$.

(C) Chávez et al.; Licensee Bentham Open.

This is an open access article licensed under the terms of the Creative Commons Attribution Non-Commercial License (http://creativecommons.org/licenses/by-nc/3.0/) which permits unrestricted, non-commercial use, distribution and reproduction in any medium, provided the work is properly cited. 